\title{
Passivity and Synchronization of Coupled Reaction-Diffusion Complex-Valued Memristive Neural Networks
}

\author{
Yanli Huang ${ }^{\mathrm{a}, *}$, Jie Hou $^{\mathrm{a}}$, Erfu Yang ${ }^{\mathrm{b}}$ \\ ${ }^{a}$ Tianjin Key Laboratory of Optoelectronic Detection Technology and System, School of Computer Science and Technology, Tiangong University, \\ Tianjin 300387, China \\ ${ }^{b}$ Department of Design, Manufacture and Engineering Management, Faculty of Engineering, University of Strathclyde, Glasgow G1 1XJ, \\ Scotland, UK
}

\begin{abstract}
This paper considers two types of coupled reaction-diffusion complex-valued memristive neural networks (CRDCVMNNs). The nodes of the first type CRDCVMNN are coupled through their state and the second one is coupled by spatial diffusion coupling term. For the former, some novel criteria for the passivity and synchronization are derived by constructing an appropriate controller and utilizing some inequality techniques as well as Lyapunov functional method. For the latter, we establish some sufficient conditions which guarantee that this type of CRDCVMNNs can realize passivity and synchronization. Finally, the effectiveness and correctness of the acquired theoretical results are verified by two numerical examples.
\end{abstract}

Keywords: State coupling, Spatial diffusion coupling, Memristive neural networks, Synchronization, Passivity

\section{Introduction}

In recent decades, coupled neural networks (CNNs) have been widely concerned because of their broad application prospects in brain science, secure communication, chaos generator design, harmonic oscillation generation and other fields. It is well known that these applications heavily depend on the dynamic behaviors of CNNs, especially the synchronization and passivity of CNNs [1, 2, 3, 4, 5, 6, 7, 8, 9, 10, 11, 12, 13, 14, 15]. In [1], the authors devoted themselves to studying the synchronization for CNNs with discontinuous activations. Several synchronization criteria for CNNs with time-varying coupling were obtained in [3]. Lin et al. [4] investigated the passivity of CNNs with different dimensional nodes by designing appropriate controllers. The authors in [14] addressed the passivity-based synchronization problem of coupled chaotic neural networks.

In 1971, Chua [16] firstly proposed the concept of memristor. Unlike resistor, the memristor can remember its past dynamic history because the memristance depends on the amount of charge passing through it. Therefore, the memristor is widely used in device modeling and signal processing, especially in simulating synaptic behavior $[17,19,18,20,21]$. In addition, by replacing the resistor with the memristor in neural networks, memristive neural networks (MNNs) can better present the neural processes in the human brain [22]. Recently, coupled memristive neural networks (CMNNs) have provoked considerable attention and a large number of interesting results on CMNNs have been reported [23, 25, 24, 26, 27, 28, 29]. In [24], the global exponential synchronization of CMNNs with time-varying delay was studied. Chen et al. [29] discussed the passivity of CMNNs with different dimensional input and output.

As we all know, complex-valued neural networks (CVNNs) are extensions of real-valued neural networks. In CVNNs, the states, connection weights and activation functions are complex-valued. Some practical problems in real world cannot be solved by real-valued neural networks but can be better solved with CVNNs. Moreover, CVNNs have a wide range of applications, including optoelectronics, emotion analysis, analogy amplification, computer version,

\footnotetext{
${ }^{*}$ Corresponding author.

Email address: huangyanli@tiangong. edu.cn (Yanli Huang)
} 
imaging and so on. Therefore, a great deal of significant papers have been published on the dynamic behaviors of CVNNs [30, 31, 32, 33]. Moreover, complex-valued MNNs (CVMNNs) can be built by replacing resistors with memristor in VLSI circuits of CVNNs as described in [34], which have a variety of different applications in image processing, engineering optimization, pattern recognition, etc. Since the actual applications of CVMNNs depend on their dynamic characteristics, it is very necessary to study the passivity and synchronization of CVMRNNs [34, 35, $36,37,38]$. The synchronization of uncertain fractional-order CVMNNs with multiple time delays was analyzed in [36]. In [38], the passivity problem for CVMNNs with time-varying delays was investigated. To our best knowledge, there are no studies on the passivity and synchronization for coupled CVMNNs.

It should be pointed out that the effect of reaction diffusion phenomena on neural networks has been neglected in the above literatures. Generally speaking, reaction diffusion phenomena in CNNs is inevitable when electrons travel in non-uniform electromagnetic fields. For example, the overall structure and dynamic behavior of cellular neural networks depend not only heavily on the evolutionary time and location (space) of each variable, but also strongly on the interaction between them, which in turn derives from the spatial distribution structure of the entire network. Therefore, taking the reaction-diffusion terms into account in CNNs is necessary, and a large number of interesting results on the synchronization and passivity of coupled reaction-diffusion neural networks (CRDNNs) have been derived [39, 40, 41, 42, 43, 44]. The synchronization and passivity problems for CRDNNs with and without parametric uncertainties were addressed in [39]. The authors put forward some conditions for guaranteeing the synchronization and passivity of CRDNNs in [40]. Nevertheless, the results on passivity and synchronization of coupled reaction-diffusion complex-valued memristive neural networks (CRDCVMNNs) have not been reported until to now. Hence, the study on synchronization and passivity of CRDCVMNNs is absolutely challenging and appealing.

It is noteworthy that great majority literatures in $[39,40,41,42,43]$ only consider the case that the network models are coupled with state. However, as far as we know, the different diffusion of each node likely have a great influence on other nodes in CRDNNs. Accordingly, the real-world networks are represented more precisely by CRDNNs with spatial diffusion coupling [45, 46, 47, 48]. In [45], the synchronization and passivity problems for CRDNNs with state and spatial diffusion couplings were addressed. Chen et al. [48] came up with several criteria for the synchronization and passivity of CRDNNs with spatial diffusion coupling and state coupling. Thus, the further study on passivity and synchronization of CRDCVMNNs with spatial diffusion coupling is worthy. It is pity that these problems have not been considered until now.

In view of the aforementioned statement, we respectively investigate the passivity and synchronization problems for CRDCVMNNs with spatial diffusion coupling and state coupling in this paper. Firstly, several criteria for ensuring synchronization and passivity of CRDCVMNNs with state coupling are established by constructing appropriate controller and employing Lyapunov functional method. Secondly, we also put forward some conditions for guaranteeing synchronization and passivity for CRDCVMNNs with spatial diffusion coupling. Finally, two examples with numerical simulations are given to verify the correctness of the results.

Accordingly, the principal goal in the present study is to investigate the passivity and synchronization of CRDCVMNNs with spatial diffusion coupling and state coupling. The main contributions of this study are as follows.

(1) The new concept of passivity for CRDCVMNNs is presented by extending the definition of the traditional passivity.

(2) The passivity and synchronization for CRDCVMNNs with state coupling are discussed, and several criteria are established by designing a state feedback controller and constructing an appropriate Lyapunov functional.

(3) Some passivity and synchronization conditions are also derived for CRDCVMNNs with spatial diffusion coupling.

The rest of this paper is organized as follows. Some notations and a lemma needed to be used throughout this paper are provided in Section 2. In Section 3, the network model of CRDCVMNNs with state coupling is presented, and then the passivity and synchronization of this kind of CRDCVMNNs are investigated. Section 4 is devoted to analyzing passivity and synchronization for CRDCVMNNs with spatial diffusion coupling. Several simulation examples are presented in Section 5 to verify the effectiveness of the obtained passivity and synchronization results. Finally, this paper is concluded in Section 6. 


\section{Preliminaries}

Notations: Let $\mathbb{R}^{N}$ and $\mathbb{C}^{N}$ be the $N$-dimensional real vector space and the $N$-dimensional complex vector space, respectively. $\lambda_{m}(\cdot), \lambda_{M}(\cdot)$ signify the minimal and the maximal eigenvalue of the corresponding matrix. Let $e=e^{R}+i e^{I}$ be a complex number, where $i$ symbols the imaginary unit, which satisfies $i=\sqrt{-1}$ and $e^{R}, e^{I} \in \mathbb{R}$ are the real and imaginary part of $e$. The norm in $\mathbb{C}^{N}$ is denoted as $\|\cdot\|$. For any vector $e(\varrho, t) \in \mathbb{C}^{N},\|e(\cdot, t)\|=\sqrt{\int_{\Omega} e^{H}(\varrho, t) e(\varrho, t) d \varrho}$ where $H$ denotes the conjugate transposition. Let $e^{R}(\varrho, t), e^{I}(\varrho, t) \in \mathbb{R}^{N}$ be the real part and imaginary part of $e(\varrho, t) \in$ $\mathbb{C}^{N}$, then one has $\|e(\cdot, t)\|=\sqrt{\int_{\Omega}\left(e^{R}(\varrho, t)\right)^{T} e^{R}(\varrho, t) d \varrho+\int_{\Omega}\left(e^{I}(\varrho, t)\right)^{T} e^{I}(\varrho, t) d \varrho}$, where $\Omega=\left\{\varrho=\left(\varrho_{1}, \varrho_{2}, \cdots, \varrho_{k}\right)^{T}|| \varrho_{q} \mid<\right.$ $\left.\xi_{q}, q=1,2, \cdots, \kappa\right\} \subset \mathbb{R}^{k}$ and $(\varrho, t) \in \Omega \times \mathbb{R}$.

Lemma 2.1. (see [49]) Let $\Omega$ be a cube $\left|\varrho_{q}\right|<\xi_{q}(q=1,2, \cdots, \kappa)$ and let $\chi(\varrho)$ be a real-valued function belonging to $C^{1}(\Omega)$ which satisfies $\left.\chi(\varrho)\right|_{\partial \Omega}=0$. Then

$$
\int_{\Omega} \chi^{2}(\varrho) d \varrho \leqslant \xi_{q}^{2} \int_{\Omega}\left(\frac{\partial \chi}{\partial \varrho}\right)_{q}^{2} d \varrho
$$

where $\varrho=\left(\varrho_{1}, \varrho_{2}, \cdots, \varrho_{\kappa}\right)^{T}$.

\section{Passivity and synchronization of CRDCVMNNs with state coupling}

\subsection{Network model}

According to the physical characteristics of memristor, a single RDCVMNN model can be described as follows:

$$
\frac{\partial y_{\iota}(\varrho, t)}{\partial t}=a_{\iota} \Delta y_{\iota}(\varrho, t)-b_{\iota} y_{\iota}(\varrho, t)+\sum_{j=1}^{n} c_{\iota j}\left(y_{\iota}(\varrho, t)\right) g_{j}\left(y_{j}\left(\varrho, t-\tau_{j}(t)\right)\right)+\sum_{j=1}^{n} d_{\iota j}\left(y_{\iota}(\varrho, t)\right) f_{j}\left(y_{j}(\varrho, t)\right),
$$

where $\iota=1,2, \cdots, n, y_{\iota}(\varrho, t)$ is the complex-valued state variable of $\iota t$ neuron; $a_{\iota}>0$ denotes the transmission diffusion coefficient along the $\iota$ th neuron; $\Delta=\sum_{q=1}^{\kappa} \frac{\partial^{2}}{\partial \varrho_{q}^{2}}$ signifies the Laplace diffusion operator on $\Omega ; b_{\iota}>0$ symbols the self-inhibition; $c_{\iota j}\left(y_{\iota}(\varrho, t)\right)$ and $d_{\iota j}\left(y_{\iota}(\varrho, t)\right)$ stand for complex-valued memristors synaptic connection weights; $g_{j}(\cdot)$ and $f_{j}(\cdot)$ present complex-valued activation functions for the delayed configuration and non-delayed one of the $j$ th neuron; the time-varying delay $\tau_{j}(t)$ satisfies $0 \leqslant \tau_{j}(t) \leqslant \tau_{j} \leqslant \tau=\max _{j=1,2, \cdots, n}\left\{\tau_{j}\right\}, \dot{\tau}_{j}(t) \leqslant \gamma_{j}<1$.

Let $y_{\iota}(\varrho, t), c_{\iota j}\left(y_{\iota}(\varrho, t)\right), d_{\iota j}\left(y_{\iota}(\varrho, t)\right), g_{j}(\cdot)$ and $f_{j}(\cdot)$ be the following with real and imaginary parts:

$$
\begin{gathered}
y_{\iota}(\varrho, t)=y_{\iota}^{R}(\varrho, t)+i y_{\iota}^{I}(\varrho, t), c_{\iota j}\left(y_{\iota}(\varrho, t)\right)=c_{\iota j}^{R}\left(y_{\iota}^{R}(\varrho, t)\right)+i c_{\iota j}^{I}\left(y_{\iota}^{I}(\varrho, t)\right), d_{\iota j}\left(y_{\iota}(\varrho, t)\right)=d_{t j}^{R}\left(y_{\iota}^{R}(\varrho, t)\right)+i d_{\iota j}^{I}\left(y_{\iota}^{I}(\varrho, t)\right), \\
f_{j}\left(y_{j}(\varrho, t)\right)=f_{j}^{R}\left(y_{j}^{R}(\varrho, t)\right)+i f_{j}^{I}\left(y_{j}^{I}(\varrho, t)\right), g_{j}\left(y_{j}\left(\varrho, t-\tau_{j}(t)\right)\right)=g_{j}^{R}\left(y_{j}^{R}\left(\varrho, t-\tau_{j}(t)\right)\right)+i g_{j}^{I}\left(y_{j}^{I}\left(\varrho, t-\tau_{j}(t)\right)\right),
\end{gathered}
$$

where $y_{\iota}^{R}(\varrho, t), c_{\iota j}^{R}\left(y_{\iota}^{R}(\varrho, t)\right), d_{\iota j}^{R}\left(y_{\iota}^{R}(\varrho, t)\right), f_{j}^{R}\left(y_{j}^{R}(\varrho, t)\right), g_{j}^{R}\left(y_{j}^{R}\left(\varrho, t-\tau_{j}(t)\right)\right)$ are the real parts of $y_{\iota}(\varrho, t), c_{\iota j}\left(y_{\iota}(\varrho, t)\right), d_{\iota j}\left(y_{\iota}(\varrho, t)\right)$, $f_{j}\left(y_{j}(\varrho, t)\right), g_{j}\left(y_{j}\left(\varrho, t-\tau_{j}(t)\right)\right)$, respectively; $i$ is the imaginary unit which satisfies $i=\sqrt{-1} ; y_{\iota}^{I}(\varrho, t), c_{\iota j}^{I}\left(y_{\iota}^{I}(\varrho, t)\right), d_{\iota j}^{I}\left(y_{\iota}^{I}(\varrho, t)\right)$, $f_{j}^{I}\left(y_{j}^{I}(\varrho, t)\right), g_{j}^{I}\left(y_{j}^{I}\left(\varrho, t-\tau_{j}(t)\right)\right)$ are the imaginary parts of $y_{\iota}(\varrho, t), c_{\iota j}\left(y_{\iota}(\varrho, t)\right), d_{\iota j}\left(y_{\iota}(\varrho, t)\right), f_{j}\left(y_{j}(\varrho, t)\right), g_{j}\left(y_{j}\left(\varrho, t-\tau_{j}(t)\right)\right)$, respectively.

In accordance with the voltage-current characteristic of memristor, one has

$$
\begin{aligned}
& c_{\iota j}^{R}\left(y_{\iota}^{R}(\varrho, t)\right)=\left\{\begin{array}{ll}
\hat{c}_{\iota j}^{R}, & \left|y_{\iota}^{R}(\varrho, t)\right| \leqslant \Gamma_{\iota}, \\
c_{\iota j}^{R}, & \left|y_{\iota}^{R}(\varrho, t)\right|>\Gamma_{\iota},
\end{array} \quad c_{\iota j}^{I}\left(y_{\iota}^{I}(\varrho, t)\right)= \begin{cases}\hat{c}_{\iota j}^{I}, & \left|y_{\iota}^{I}(\varrho, t)\right| \leqslant \Gamma_{\iota}, \\
\check{c}_{\iota j}^{I}, & \left|y_{\iota}^{I}(\varrho, t)\right|>\Gamma_{\iota},\end{cases} \right. \\
& d_{\iota j}^{R}\left(y_{\iota}^{R}(\varrho, t)\right)=\left\{\begin{array}{ll}
\hat{d}_{\iota j}^{R}, & \left|y_{\iota}^{R}(\varrho, t)\right| \leqslant \Gamma_{\iota}, \\
\check{d}_{\iota j}^{R}, & \left|y_{\iota}^{R}(\varrho, t)\right|>\Gamma_{\iota},
\end{array} \quad d_{\iota j}^{I}\left(y_{\iota}^{I}(\varrho, t)\right)= \begin{cases}\hat{d}_{\iota j}^{I}, & \left|y_{\iota}^{I}(\varrho, t)\right| \leqslant \Gamma_{\iota}, \\
\check{d}_{\iota j}^{I}, & \left|y_{\iota}^{I}(\varrho, t)\right|>\Gamma_{\iota},\end{cases} \right.
\end{aligned}
$$

where $\iota, j \in\{1,2, \cdots, n\} ; \hat{c}_{\iota j}^{R}, \hat{c}_{\iota j}^{I}, \breve{c}_{\iota j}^{R}, \breve{c}_{\iota j}^{I}, \hat{d}_{\iota j}^{R}, \hat{d}_{\iota j}^{I}, \breve{d}_{\iota j}^{R}, \breve{d}_{\iota j}^{I}$ are all constants; $\Gamma_{\iota}>0$ symbols the threshold level. Denote

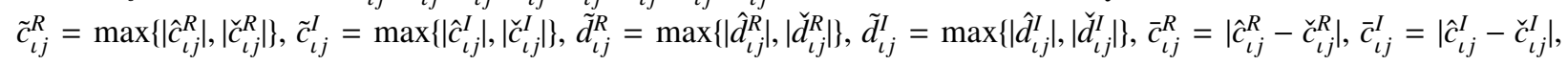


$\bar{d}_{\iota j}^{R}=\left|\hat{d}_{\iota j}^{R}-\breve{d}_{\iota j}^{R}\right|, \bar{d}_{\iota j}^{I}=\left|\hat{d}_{\iota j}^{I}-\breve{d}_{\iota j}^{I}\right|, \bar{C}^{R}=\left(\bar{c}_{\iota j}^{R}\right)_{n \times n}, \bar{C}^{I}=\left(\bar{c}_{\iota j}^{I}\right)_{n \times n}, \tilde{C}^{R}=\operatorname{diag}\left(\sum_{j=1}^{n}\left(\tilde{c}_{1 j}^{R}\right)^{2}, \sum_{j=1}^{n}\left(\tilde{c}_{2 j}^{R}\right)^{2}, \cdots, \sum_{j=1}^{n}\left(\tilde{c}_{n j}^{R}\right)^{2}\right), \tilde{C}^{I}=$ $\operatorname{diag}\left(\sum_{j=1}^{n}\left(\tilde{c}_{1 j}^{I}\right)^{2}, \sum_{j=1}^{n}\left(\tilde{c}_{2 j}^{I}\right)^{2}, \cdots, \sum_{j=1}^{n}\left(\tilde{c}_{n j}^{I}\right)^{2}\right), \bar{D}^{R}=\left(\bar{d}_{l j}^{R}\right)_{n \times n}, \bar{D}^{I}=\left(\bar{d}_{l j}^{I}\right)_{n \times n}, \tilde{D}^{R}=\operatorname{diag}\left(\sum_{j=1}^{n}\left(\tilde{d}_{1 j}^{R}\right)^{2}, \sum_{j=1}^{n}\left(\tilde{d}_{2 j}^{R}\right)^{2}, \cdots, \sum_{j=1}^{n}\left(\tilde{d}_{n j}^{R}\right)^{2}\right)$, $\tilde{D}^{I}=\operatorname{diag}\left(\sum_{j=1}^{n}\left(\tilde{d}_{1 j}^{I}\right)^{2}, \sum_{j=1}^{n}\left(\tilde{d}_{2 j}^{I}\right)^{2}, \cdots, \sum_{j=1}^{n}\left(\tilde{d}_{n j}^{I}\right)^{2}\right)$.

According to the above description, the network (1) can be separated into real and imaginary parts as follows:

$$
\begin{aligned}
\frac{\partial y_{\iota}^{R}(\varrho, t)}{\partial t}= & a_{\iota} \Delta y_{\iota}^{R}(\varrho, t)-b_{\iota} y_{\iota}^{R}(\varrho, t)+\sum_{j=1}^{n} c_{\iota j}^{R}\left(y_{\iota}^{R}(\varrho, t)\right) g_{j}^{R}\left(y_{j}^{R}\left(\varrho, t-\tau_{j}(t)\right)\right)+\sum_{j=1}^{n} d_{\iota j}^{R}\left(y_{\iota}^{R}(\varrho, t)\right) f_{j}^{R}\left(y_{j}^{R}(\varrho, t)\right) \\
& -\sum_{j=1}^{n} d_{\iota j}^{I}\left(y_{\iota}^{I}(\varrho, t)\right) f_{j}^{I}\left(y_{j}^{I}(\varrho, t)\right)-\sum_{j=1}^{n} c_{\iota j}^{I}\left(y_{\iota}^{I}(\varrho, t)\right) g_{j}^{I}\left(y_{j}^{I}\left(\varrho, t-\tau_{j}(t)\right)\right), \\
\frac{\partial y_{\iota}^{I}(\varrho, t)}{\partial t}= & a_{\iota} \Delta y_{\iota}^{I}(\varrho, t)-b_{\iota} y_{\iota}^{I}(\varrho, t)+\sum_{j=1}^{n} c_{\iota j}^{R}\left(y_{\iota}^{R}(\varrho, t)\right) g_{j}^{I}\left(y_{j}^{I}\left(\varrho, t-\tau_{j}(t)\right)\right)+\sum_{j=1}^{n} d_{\iota j}^{R}\left(y_{\iota}^{R}(\varrho, t)\right) f_{j}^{I}\left(y_{j}^{I}(\varrho, t)\right) \\
& +\sum_{j=1}^{n} d_{\iota j}^{I}\left(y_{\iota}^{I}(\varrho, t)\right) f_{j}^{R}\left(y_{j}^{R}(\varrho, t)\right)+\sum_{j=1}^{n} c_{\iota j}^{I}\left(y_{\iota}^{I}(\varrho, t)\right) g_{j}^{R}\left(y_{j}^{R}\left(\varrho, t-\tau_{j}(t)\right)\right) .
\end{aligned}
$$

In this section, we consider the following CRDCVMNNs consisting of $N$ RDCVMNNs (1):

$$
\begin{aligned}
\frac{\partial Y_{s}(\varrho, t)}{\partial t}= & A \Delta Y_{s}(\varrho, t)-B Y_{s}(\varrho, t)+C\left(Y_{s}(\varrho, t)\right) g\left(\overline{Y_{s}(\varrho, t)}\right)+u_{s}(\varrho, t)+D\left(Y_{s}(\varrho, t)\right) f\left(Y_{s}(\varrho, t)\right) \\
& +h \sum_{\kappa=1}^{N} H_{s \kappa} M Y_{\kappa}(\varrho, t)+x_{s}(\varrho, t),
\end{aligned}
$$

where $s=1,2, \cdots, N, Y_{s}(\varrho, t)=\left(Y_{s 1}(\varrho, t), Y_{s 2}(\varrho, t), \cdots, Y_{s n}(\varrho, t)\right) \in \mathbb{C}^{n}$ represents the complex-valued state variable of the sth node; $A=\operatorname{diag}\left(a_{1}, a_{2}, \cdots, a_{n}\right) \in \mathbb{R}^{n \times n}>0,0<B=\operatorname{diag}\left(b_{1}, b_{2}, \cdots, b_{n}\right) \in \mathbb{R}^{n \times n} ; \overline{Y_{s}(\varrho, t)}=\left(Y_{s 1}(\varrho, t-\right.$ $\left.\left.\tau_{1}(t)\right), Y_{s 2}\left(\varrho, t-\tau_{2}(t)\right), \cdots, Y_{s n}\left(\varrho, t-\tau_{n}(t)\right)\right)^{T} \in \mathbb{C}^{n} ; g\left(\overline{Y_{s}(\varrho, t)}\right)=\left(g_{1}\left(Y_{s 1}\left(\varrho, t-\tau_{1}(t)\right)\right), g_{2}\left(Y_{s 2}\left(\varrho, t-\tau_{2}(t)\right)\right), \cdots, g_{n}\left(Y_{s n}(\varrho, t-\right.\right.$ $\left.\left.\left.\tau_{n}(t)\right)\right)\right)^{T} \in \mathbb{C}^{n} ; f\left(Y_{s}(\varrho, t)\right)=\left(f_{1}\left(Y_{s 1}(\varrho, t)\right), f_{2}\left(Y_{s 2}(\varrho, t)\right), \cdots, f_{n}\left(Y_{s n}(\varrho, t)\right)\right)^{T} \in \mathbb{C}^{n} ; M \in \mathbb{R}^{n \times n}$ stands for the inner coupling matrix; $C\left(Y_{s}(\varrho, t)\right)=\left(c_{\iota j}\left(Y_{s l}(\varrho, t)\right)\right)_{n \times n} \in \mathbb{C}^{n \times n}, D\left(Y_{s}(\varrho, t)\right)=\left(d_{\iota j}\left(Y_{s l}(\varrho, t)\right)\right)_{n \times n} \in \mathbb{C}^{n \times n}$, where $\iota, j=1,2, \cdots, n$; $u_{s}(\varrho, t)=u_{s}^{R}(\varrho, t)+i u_{s}^{I}(\varrho, t)=\left(u_{s 1}(\varrho, t), u_{s 2}(\varrho, t), \cdots, u_{s n}(\varrho, t)\right)^{T} \in \mathbb{C}^{n}$ is the controller to be designed for obtaining a certain control objective; $x_{s}(\varrho, t)=x_{s}^{R}(\varrho, t)+i x_{s}^{I}(\varrho, t)=\left(x_{s 1}(\varrho, t), x_{s 2}(\varrho, t), \cdots, x_{s n}(\varrho, t)\right)^{T} \in \mathbb{C}^{n}$ denotes the external input of the network; $h>0$ is the overall coupling strength; $H=\left(H_{S K}\right)_{N \times N}$ expresses coupling weight between nodes, where $H_{s \kappa}=H_{\kappa s}>0$ if and only if there exists a connection between node s and node $\kappa$; if not, $H_{s \kappa}=H_{\kappa s}=0(s \neq \kappa)$; and

$$
H_{s s}=-\sum_{\substack{k=1 \\ k \neq s}}^{N} H_{s k}, s=1,2, \cdots, N .
$$

For network (2),

$$
\begin{aligned}
& Y_{s}(\varrho, t)=\phi_{s}(\varrho, t) \in \mathbb{C}^{n}, \quad(\varrho, t) \in \Omega \times[-\tau, 0], \\
& Y_{s}(\varrho, t)=0, \quad(\varrho, t) \in \partial \Omega \times[-\tau,+\infty),
\end{aligned}
$$

where $\phi_{s}(\varrho, t)=\left(\phi_{s 1}(\varrho, t), \phi_{s 2}(\varrho, t), \cdots, \phi_{s n}(\varrho, t)\right)^{T} \in \mathbb{C}^{n}$ is bounded and continuous on $\Omega \times[-\tau, 0]$.

Then, the network (2) can be separated into real and imaginary parts as follows:

$$
\begin{aligned}
\frac{\partial Y_{s}^{R}(\varrho, t)}{\partial t}= & A \triangle Y_{s}^{R}(\varrho, t)-B Y_{s}^{R}(\varrho, t)+C^{R}\left(Y_{s}^{R}(\varrho, t)\right) g^{R}\left(\overline{Y_{s}^{R}(\varrho, t)}\right)+u_{s}^{R}(\varrho, t)-C^{I}\left(Y_{s}^{I}(\varrho, t)\right) g^{I}\left(\overline{Y_{s}^{I}(\varrho, t)}\right) \\
& +D^{R}\left(Y_{s}^{R}(\varrho, t)\right) f^{R}\left(Y_{s}^{R}(\varrho, t)\right)+x_{s}^{R}(\varrho, t)-D^{I}\left(Y_{s}^{I}(\varrho, t)\right) f^{I}\left(Y_{s}^{I}(\varrho, t)\right)+h \sum_{k=1}^{N} H_{s k} M Y_{\kappa}^{R}(\varrho, t), \\
\frac{\partial Y_{s}^{I}(\varrho, t)}{\partial t}= & \left.\left.A \triangle Y_{s}^{I}(\varrho, t)-B Y_{s}^{I}(\varrho, t)+C^{R}\left(Y_{s}^{R}(\varrho, t)\right) g^{I} \overline{\left(Y_{s}^{I}(\varrho, t)\right.}\right)+u_{s}^{I}(\varrho, t)+C^{I}\left(Y_{s}^{I}(\varrho, t)\right) g^{R} \overline{Y_{s}^{R}(\varrho, t)}\right)
\end{aligned}
$$




$$
+D^{R}\left(Y_{s}^{R}(\varrho, t)\right) f^{I}\left(Y_{s}^{I}(\varrho, t)\right)+x_{s}^{I}(\varrho, t)+D^{I}\left(Y_{s}^{I}(\varrho, t)\right) f^{R}\left(Y_{s}^{R}(\varrho, t)\right)+h \sum_{k=1}^{N} H_{s k} M Y_{\kappa}^{I}(\varrho, t),
$$

where

$$
\begin{gathered}
C^{R}(\cdot)=\left(c_{t i}^{R}(\cdot)\right)_{n \times n}, D^{R}(\cdot)=\left(d_{t j}^{R}(\cdot)\right)_{n \times n}, C^{I}(\cdot)=\left(c_{t j}^{I}(\cdot)\right)_{n \times n}, D^{I}(\cdot)=\left(d_{t j}^{I}(\cdot)\right)_{n \times n}, \\
g^{R} \overline{\left(Y_{s}^{R}(\varrho, t)\right)}=\left(g_{1}^{R}\left(Y_{s 1}^{R}\left(\varrho, t-\tau_{1}(t)\right)\right), g_{2}^{R}\left(Y_{s 2}^{R}\left(\varrho, t-\tau_{2}(t)\right)\right), \cdots, g_{n}^{R}\left(Y_{s n}^{R}\left(\varrho, t-\tau_{n}(t)\right)\right)\right)^{T}, \\
g^{I}\left(\overline{Y_{s}^{I}(\varrho, t)}\right)=\left(g_{1}^{I}\left(Y_{s 1}^{I}\left(\varrho, t-\tau_{1}(t)\right)\right), g_{2}^{I}\left(Y_{s 2}^{I}\left(\varrho, t-\tau_{2}(t)\right)\right), \cdots, g_{n}^{I}\left(Y_{s n}^{I}\left(\varrho, t-\tau_{n}(t)\right)\right)\right)^{T}, \\
f^{R}\left(Y_{s}^{R}(\varrho, t)\right)=\left(f_{1}^{R}\left(Y_{s 1}^{R}(\varrho, t)\right), f_{2}^{R}\left(Y_{s 2}^{R}(\varrho, t)\right), \cdots, f_{n}^{R}\left(Y_{s n}^{R}(\varrho, t)\right)\right)^{T}, \\
f^{I}\left(Y_{s}^{I}(\varrho, t)\right)=\left(f_{1}^{I}\left(Y_{s 1}^{I}(\varrho, t)\right), f_{2}^{I}\left(Y_{s 2}^{I}(\varrho, t)\right), \cdots, f_{n}^{I}\left(Y_{s n}^{I}(\varrho, t)\right)\right)^{T}, \\
Y_{s}^{R}(\varrho, t)=\left(Y_{s 1}^{R}(\varrho, t), Y_{s 2}^{R}(\varrho, t), \cdots, Y_{s n}^{R}(\varrho, t)\right)^{T}, \\
Y_{s}^{I}(\varrho, t)=\left(Y_{s 1}^{I}(\varrho, t), Y_{s 2}^{I}(\varrho, t), \cdots, Y_{s n}^{I}(\varrho, t)\right)^{T}, \\
u_{s}^{R}(\varrho, t)=\left(u_{s 1}^{R}(\varrho, t), u_{s 2}^{R}(\varrho, t), \cdots, u_{s n}^{R}(\varrho, t)\right)^{T}, \\
u_{s}^{I}(\varrho, t)=\left(u_{s 1}^{I}(\varrho, t), u_{s 2}^{I}(\varrho, t), \cdots, u_{s n}^{I}(\varrho, t)\right)^{T}, \\
x_{s}^{R}(\varrho, t)=\left(x_{s 1}^{R}(\varrho, t), x_{s 2}^{R}(\varrho, t), \cdots, x_{s n}^{R}(\varrho, t)\right)^{T}, \\
x_{s}^{I}(\varrho, t)=\left(x_{s 1}^{I}(\varrho, t), x_{s 2}^{I}(\varrho, t), \cdots, x_{s n}^{I}(\varrho, t)\right)^{T} .
\end{gathered}
$$

For network (3),

$$
\begin{aligned}
& Y_{s}^{R}(\varrho, t)=\phi_{s}^{R}(\varrho, t) \in \mathbb{R}^{n}, \quad(\varrho, t) \in \Omega \times[-\tau, 0], \\
& Y_{s}^{R}(\varrho, t)=0, \quad(\varrho, t) \in \partial \Omega \times[-\tau,+\infty),
\end{aligned}
$$

where $\phi_{s}^{R}(\varrho, t)=\left(\phi_{s 1}^{R}(\varrho, t), \phi_{s 2}^{R}(\varrho, t), \cdots, \phi_{s n}^{R}(\varrho, t)\right)^{T} \in \mathbb{R}^{n}$ is bounded and continuous on $\Omega \times[-\tau, 0]$.

For network (4),

$$
\begin{aligned}
& Y_{s}^{I}(\varrho, t)=\phi_{s}^{I}(\varrho, t) \in \mathbb{R}^{n}, \quad(\varrho, t) \in \Omega \times[-\tau, 0], \\
& Y_{s}^{I}(\varrho, t)=0, \quad(\varrho, t) \in \partial \Omega \times[-\tau,+\infty),
\end{aligned}
$$

where $\phi_{s}^{I}(\varrho, t)=\left(\phi_{s 1}^{I}(\varrho, t), \phi_{s 2}^{I}(\varrho, t), \cdots, \phi_{s n}^{I}(\varrho, t)\right)^{T} \in \mathbb{R}^{n}$ is continuous and bounded on $\Omega \times[-\tau, 0]$.

Assumption 1. (see [29]) Throughout this paper, we assume that the real part $f_{s}^{R}(\cdot)$ and the imaginary part $f_{s}^{I}(\cdot)$ of function $f_{s}(\cdot)$ and the real part $g_{s}^{R}(\cdot)$ and the imaginary part $g_{s}^{I}(\cdot)$ of function $g_{s}(\cdot)$ satisfy the following inequalities for any $\alpha_{1}, \alpha_{2} \in \mathbb{R}$ :

$$
\begin{aligned}
& \left|f_{s}^{R}(\cdot)\right| \leqslant F_{s}^{R},\left|f_{s}^{I}(\cdot)\right| \leqslant F_{s}^{I},\left|g_{s}^{R}(\cdot)\right| \leqslant G_{s}^{R},\left|g_{s}^{I}(\cdot)\right| \leqslant G_{s}^{I}, \\
& \left|f_{s}^{R}\left(\alpha_{1}\right)-f_{s}^{R}\left(\alpha_{2}\right)\right| \leqslant l_{s}^{R}\left|\alpha_{1}-\alpha_{2}\right|,\left|f_{s}^{I}\left(\alpha_{1}\right)-f_{s}^{I}\left(\alpha_{2}\right)\right| \leqslant l_{s}^{I}\left|\alpha_{1}-\alpha_{2}\right|, \\
& \left|g_{s}^{R}\left(\alpha_{1}\right)-g_{s}^{R}\left(\alpha_{2}\right)\right| \leqslant \eta_{s}^{R}\left|\alpha_{1}-\alpha_{2}\right|,\left|g_{s}^{I}\left(\alpha_{1}\right)-g_{s}^{I}\left(\alpha_{2}\right)\right| \leqslant \eta_{s}^{I}\left|\alpha_{1}-\alpha_{2}\right|,
\end{aligned}
$$

where $F_{s}^{R}, F_{s}^{I}, G_{s}^{R}, G_{s}^{I}, l_{s}^{R}, l_{s}^{I}, \eta_{s}^{R}, \eta_{s}^{I}$ are positive constants.

Suppose $Y_{0}(\varrho, t)=\left(Y_{01}(\varrho, t), Y_{02}(\varrho, t), \cdots, Y_{0 n}(\varrho, t)\right)^{T} \in \mathbb{C}^{n}$ is an arbitrary solution of the network (2), then

$$
\frac{\partial Y_{0}(\varrho, t)}{\partial t}=A \Delta Y_{0}(\varrho, t)-B Y_{0}(\varrho, t)+C\left(Y_{0}(\varrho, t)\right) g\left(Y_{0}(\varrho, t)\right)+D\left(Y_{0}(\varrho, t)\right) f\left(Y_{0}(\varrho, t)\right),
$$

where $Y_{0}(\varrho, t)=Y_{0}^{R}(\varrho, t)+i Y_{0}^{I}(\varrho, t)$. Then, we can separate (5) into real and imaginary parts as follows:

$$
\frac{\partial Y_{0}^{R}(\varrho, t)}{\partial t}=A \triangle Y_{0}^{R}(\varrho, t)-B Y_{0}^{R}(\varrho, t)+C^{R}\left(Y_{0}^{R}(\varrho, t)\right) g^{R}\left(Y_{0}^{R}(\varrho, t)\right)-C^{I}\left(Y_{0}^{I}(\varrho, t)\right) g^{I}\left(Y_{0}^{I}(\varrho, t)\right)+D^{R}\left(Y_{0}^{R}(\varrho, t)\right) f^{R}\left(Y_{0}^{R}(\varrho, t)\right)
$$




$$
\begin{aligned}
& -D^{I}\left(Y_{0}^{I}(\varrho, t)\right) f^{I}\left(Y_{0}^{I}(\varrho, t)\right), \\
\frac{\partial Y_{0}^{I}(\varrho, t)}{\partial t}= & A \triangle Y_{0}^{I}(\varrho, t)-B Y_{0}^{I}(\varrho, t)+C^{R}\left(Y_{0}^{R}(\varrho, t)\right) g^{I}\left(Y_{0}^{I}(\varrho, t)\right)+C^{I}\left(Y_{0}^{I}(\varrho, t)\right) g^{R}\left(Y_{0}^{R}(\varrho, t)\right)+D^{R}\left(Y_{0}^{R}(\varrho, t)\right) f^{I}\left(Y_{0}^{I}(\varrho, t)\right) \\
& +D^{I}\left(Y_{0}^{I}(\varrho, t)\right) f^{R}\left(Y_{0}^{R}(\varrho, t)\right) .
\end{aligned}
$$

Let $e_{s}(\varrho, t)=Y_{s}(\varrho, t)-Y_{0}(\varrho, t)$, then

$$
\begin{aligned}
\frac{\partial e_{s}(\varrho, t)}{\partial t}= & A \Delta e_{s}(\varrho, t)-B e_{s}(\varrho, t)+C\left(Y_{s}(\varrho, t)\right) g\left(\overline{Y_{s}(\varrho, t)}\right)+x_{s}(\varrho, t)-C\left(Y_{0}(\varrho, t)\right) g\left(Y_{0}(\varrho, t)\right)+D\left(Y_{s}(\varrho, t)\right) f\left(Y_{s}(\varrho, t)\right) \\
& +u_{s}(\varrho, t)-D\left(Y_{0}(\varrho, t)\right) f\left(Y_{0}(\varrho, t)\right)+h \sum_{k=1}^{N} H_{s k} M e_{\kappa}(\varrho, t),
\end{aligned}
$$

where $s=1,2, \cdots, N$ and $e_{s}(\varrho, t)=\left(e_{s 1}(\varrho, t), e_{s 2}(\varrho, t), \cdots, e_{s n}(\varrho, t)\right)^{T}$.

By separating (6) into real and imaginary parts, we have

$$
\begin{aligned}
\frac{\partial e_{s}^{R}(\varrho, t)}{\partial t}= & A \triangle e_{s}^{R}(\varrho, t)-B e_{s}^{R}(\varrho, t)+D^{R}\left(Y_{s}^{R}(\varrho, t)\right) P^{R}\left(e_{s}^{R}(\varrho, t)\right)+x_{s}^{R}(\varrho, t)-D^{I}\left(Y_{s}^{I}(\varrho, t)\right) P^{I}\left(e_{s}^{I}(\varrho, t)\right)+C^{R}\left(Y_{s}^{R}(\varrho, t)\right) Q^{R}\left(\overline{e_{s}^{R}(\varrho, t)}\right) \\
& +u_{s}^{R}(\varrho, t)-C^{I}\left(Y_{s}^{I}(\varrho, t)\right) Q^{I}\left(\overline{e_{s}^{I}(\varrho, t)}\right)+h \sum_{k=1}^{N} H_{s k} M e_{\kappa}^{R}(\varrho, t)+\left(D^{R}\left(Y_{s}^{R}(\varrho, t)\right)-D^{R}\left(Y_{0}^{R}(\varrho, t)\right)\right) f^{R}\left(Y_{0}^{R}(\varrho, t)\right)-\left(D^{I}\left(Y_{s}^{I}(\varrho, t)\right)\right. \\
& \left.-D^{I}\left(Y_{0}^{I}(\varrho, t)\right)\right) f^{I}\left(Y_{0}^{I}(\varrho, t)\right)+\left(C^{R}\left(Y_{s}^{R}(\varrho, t)\right)-C^{R}\left(Y_{0}^{R}(\varrho, t)\right)\right) g^{R}\left(Y_{0}^{R}(\varrho, t)\right)-\left(C^{I}\left(Y_{s}^{I}(\varrho, t)\right)-C^{I}\left(Y_{0}^{I}(\varrho, t)\right)\right) g^{I}\left(Y_{0}^{I}(\varrho, t)\right), \\
\frac{\partial e_{s}^{I}(\varrho, t)}{\partial t}= & A \triangle e_{s}^{I}(\varrho, t)-B e_{s}^{I}(\varrho, t)+D^{R}\left(Y_{s}^{R}(\varrho, t)\right) P^{I}\left(e_{s}^{I}(\varrho, t)\right)+x_{s}^{I}(\varrho, t)+D^{I}\left(Y_{s}^{I}(\varrho, t)\right) P^{R}\left(e_{s}^{R}(\varrho, t)\right)+C^{R}\left(Y_{s}^{R}(\varrho, t)\right) Q^{I}\left(\overline{\left.e_{s}^{I}(\varrho, t)\right)}\right. \\
& +u_{s}^{I}(\varrho, t)+C^{I}\left(Y_{s}^{I}(\varrho, t)\right) Q^{R}\left(\overline{\left(e_{s}^{R}(\varrho, t)\right)}\right)+h \sum_{k=1}^{N} H_{s k} M e_{\kappa}^{I}(\varrho, t)+\left(D^{R}\left(Y_{s}^{R}(\varrho, t)\right)-D^{R}\left(Y_{0}^{R}(\varrho, t)\right)\right) f^{I}\left(Y_{0}^{I}(\varrho, t)\right)+\left(D^{I}\left(Y_{s}^{I}(\varrho, t)\right)\right. \\
& \left.-D^{I}\left(Y_{0}^{I}(\varrho, t)\right)\right) f^{R}\left(Y_{0}^{R}(\varrho, t)\right)+\left(C^{R}\left(Y_{s}^{R}(\varrho, t)\right)-C^{R}\left(Y_{0}^{R}(\varrho, t)\right)\right) g^{I}\left(Y_{0}^{I}(\varrho, t)\right)+\left(C^{I}\left(Y_{s}^{I}(\varrho, t)\right)-C^{I}\left(Y_{0}^{I}(\varrho, t)\right)\right) g^{R}\left(Y_{0}^{R}(\varrho, t)\right),
\end{aligned}
$$

where $e_{s}^{R}(\varrho, t)=\left(e_{s 1}^{R}(\varrho, t), e_{s 2}^{R}(\varrho, t), \cdots, e_{s n}^{R}(\varrho, t)\right)^{T}, e_{s}^{I}(\varrho, t)=\left(e_{s 1}^{I}(\varrho, t), e_{s 2}^{I}(\varrho, t), \cdots, e_{s n}^{I}(\varrho, t)\right)^{T}, \overline{e_{s}^{R}(\varrho, t)}=\left(e_{s 1}^{R}(\varrho, t-\right.$ $\left.\left.\tau_{1}(t)\right), e_{s 2}^{R}\left(\varrho, t-\tau_{2}(t)\right), \cdots, e_{s n}^{R}\left(\varrho, t-\tau_{n}(t)\right)\right)^{T}, \overline{e_{s}^{I}(\varrho, t)}=\left(e_{s 1}^{I}\left(\varrho, t-\tau_{1}(t)\right), e_{s 2}^{I}\left(\varrho, t-\tau_{2}(t)\right), \cdots, e_{s n}^{I}\left(\varrho, t-\tau_{n}(t)\right)\right)^{T}, P^{R}\left(e_{s}^{R}(\varrho, t)\right)=$ $f^{R}\left(Y_{s}^{R}(\varrho, t)\right)-f^{R}\left(Y_{0}^{R}(\varrho, t)\right), P^{I}\left(e_{s}^{I}(\varrho, t)\right)=f^{I}\left(Y_{s}^{I}(\varrho, t)\right)-f^{I}\left(Y_{0}^{I}(\varrho, t)\right), Q^{R}\left(\overline{e_{s}^{R}(\varrho, t)}\right)=g^{R}\left(\overline{Y_{s}^{R}(\varrho, t)}\right)-g^{R}\left(Y_{0}^{R}(\varrho, t)\right)$ and $\left.Q^{I} \overline{\left(e_{s}^{I}(\varrho, t)\right.}\right)=g^{I}\left(\overline{Y_{s}^{I}(\varrho, t)}\right)-g^{I}\left(Y_{0}^{I}(\varrho, t)\right)$.

Definition 3.1. (see [44]) If there exists a constant $\rho>0$ satisfying the following inequality:

$$
\int_{t_{1}}^{t_{2}} \int_{\Omega}\left[\left(z^{R}(\varrho, t)\right)^{T} x^{R}(\varrho, t)+\left(z^{I}(\varrho, t)\right)^{T} x^{I}(\varrho, t)\right] d \varrho d t \geqslant V\left(t_{2}\right)-V\left(t_{1}\right)-\rho \int_{t_{1}}^{t_{2}} \int_{\Omega}\left[\left(x^{R}(\varrho, t)\right)^{T} x^{R}(\varrho, t)+\left(x^{I}(\varrho, t)\right)^{T} x^{I}(\varrho, t)\right] d \varrho d t
$$

for any $t_{2}, t_{1} \in \mathbb{R}^{+}$and $t_{2} \geqslant t_{1}$, where $V(t): \mathbb{R}^{+} \rightarrow \mathbb{R}^{+}$is the storage function, then the network (6) is said to be passive.

Definition 3.2. (see [48]) The network (2) is synchronized if

$$
\lim _{t \rightarrow \infty}\left\|Y_{s}(\cdot, t)-Y_{0}(\cdot, t)\right\|=0, \quad s=1,2, \cdots, N,
$$

under the condition $x_{s}(\varrho, t)=0, s=1,2, \cdots, N$.

\subsection{Passivity control}

For the network (2), we design the state feedback controller as follows:

$$
\left\{\begin{array}{l}
u_{s}^{R}(\varrho, t)=-\Upsilon^{R} e_{s}^{R}(\varrho, t)-\operatorname{sign}\left(e_{s}^{R}(\varrho, t)\right)\left(\bar{D}^{R} \bar{F}^{R}+\bar{D}^{I} \bar{F}^{I}+\bar{C}^{R} \bar{G}^{R}+\bar{C}^{I} \bar{G}^{I}\right), \\
u_{s}^{I}(\varrho, t)=-\Upsilon^{I} e_{s}^{I}(\varrho, t)-\operatorname{sign}\left(e_{s}^{I}(\varrho, t)\right)\left(\bar{D}^{R} \bar{F}^{I}+\bar{D}^{I} \bar{F}^{R}+\bar{C}^{R} \bar{G}^{I}+\bar{C}^{I} \bar{G}^{R}\right),
\end{array}\right.
$$


where $s=1,2, \cdots, N, \Upsilon^{R}=\operatorname{diag}\left(v_{1}^{R}, v_{2}^{R}, \cdots, v_{n}^{R}\right) \in \mathbb{R}^{n \times n}$ and $\Upsilon^{I}=\operatorname{diag}\left(v_{1}^{I}, v_{2}^{I}, \cdots, v_{n}^{I}\right) \in \mathbb{R}^{n \times n}$ are the positive definite controller gain matrices, $\mathbb{R}^{+} \ni v_{\iota}^{R}>0$ and $\mathbb{R}^{+} \ni v_{\iota}^{I}>0 ; \bar{F}^{R}=\left(F_{1}^{R}, F_{1}^{R}, \cdots, F_{n}^{R}\right)^{T}, \bar{F}^{I}=\left(F_{1}^{I}, F_{1}^{I}, \cdots, F_{n}^{I}\right)^{T}, \bar{G}^{R}=$ $\left(G_{1}^{R}, G_{1}^{R}, \cdots, G_{n}^{R}\right)^{T}$ and $\bar{G}^{I}=\left(G_{1}^{I}, G_{1}^{I}, \cdots, G_{n}^{I}\right)^{T} ; \operatorname{sign}\left(e_{s}^{R}(\varrho, t)\right)=\operatorname{diag}\left(\operatorname{sign}\left(e_{s 1}^{R}(\varrho, t)\right), \operatorname{sign}\left(e_{s 2}^{R}(\varrho, t)\right), \cdots, \operatorname{sign}\left(e_{s n}^{R}(\varrho, t)\right)\right)$ and $\operatorname{sign}\left(e_{s}^{I}(\varrho, t)\right)=\operatorname{diag}\left(\operatorname{sign}\left(e_{s 1}^{I}(\varrho, t)\right), \operatorname{sign}\left(e_{s 2}^{I}(\varrho, t)\right), \cdots, \operatorname{sign}\left(e_{s n}^{I}(\varrho, t)\right)\right)$.

The output vector $z_{s}(\varrho, t) \in \mathbb{C}^{n}$ of the system (6) is described as follows:

$$
z_{s}(\varrho, t)=K_{1} e_{s}(\varrho, t)+K_{2} x_{s}(\varrho, t),
$$

where $K_{1} \in \mathbb{R}^{n \times n}$ and $K_{2} \in \mathbb{R}^{n \times n}$.

For convenience, we denote

$$
\begin{aligned}
& L^{R}=\operatorname{diag}\left(\left(l_{1}^{R}\right)^{2},\left(l_{2}^{R}\right)^{2}, \cdots,\left(l_{n}^{R}\right)^{2}\right), L^{I}=\operatorname{diag}\left(\left(l_{1}^{I}\right)^{2},\left(l_{2}^{I}\right)^{2}, \cdots,\left(l_{n}^{I}\right)^{2}\right), \\
& \zeta^{R}=\operatorname{diag}\left(\left(\eta_{1}^{R}\right)^{2},\left(\eta_{2}^{R}\right)^{2}, \cdots,\left(\eta_{n}^{R}\right)^{2}\right), \zeta^{I}=\operatorname{diag}\left(\left(\eta_{1}^{I}\right)^{2},\left(\eta_{2}^{I}\right)^{2}, \cdots,\left(\eta_{n}^{I}\right)^{2}\right), \\
& e^{R}(\varrho, t)=\left(\left(e_{1}^{R}(\varrho, t)\right)^{T},\left(e_{2}^{R}(\varrho, t)\right)^{T}, \cdots,\left(e_{N}^{R}(\varrho, t)\right)^{T}\right)^{T}, \\
& e^{I}(\varrho, t)=\left(\left(e_{1}^{I}(\varrho, t)\right)^{T},\left(e_{2}^{I}(\varrho, t)\right)^{T}, \cdots,\left(e_{N}^{I}(\varrho, t)\right)^{T}\right)^{T}, \\
& \overline{e^{R}(\varrho, t)}=\left(\left(\overline{e_{1}^{R}(\varrho, t)}\right)^{T},\left(\overline{e_{2}^{R}(\varrho, t)}\right)^{T}, \cdots,\left(\overline{e_{N}^{R}(\varrho, t)}\right)^{T}\right)^{T}, \\
& \overline{e^{I}(\varrho, t)}=\left(\left(\overline{e_{1}^{I}(\varrho, t)}\right)^{T},\left(\overline{e_{2}^{I}(\varrho, t)}\right)^{T}, \cdots,\left(\overline{e_{N}^{I}(\varrho, t)}\right)^{T}\right)^{T}, \\
& x(\varrho, t)=\left(x_{1}^{H}(\varrho, t), x_{2}^{H}(\varrho, t), \cdots, x_{N}^{H}(\varrho, t)\right)^{H}, \\
& z(\varrho, t)=\left(z_{1}^{H}(\varrho, t), z_{2}^{H}(\varrho, t), \cdots, z_{N}^{H}(\varrho, t)\right)^{H}, \\
& \Gamma=\operatorname{diag}\left(\frac{1}{1-\gamma_{1}}, \frac{1}{1-\gamma_{2}}, \cdots, \frac{1}{1-\gamma_{n}}\right) .
\end{aligned}
$$

Theorem 3.1. If there exists a constant $\rho>0$ such that

$$
\left(\begin{array}{cc}
\Psi_{1}^{R} & \Xi^{R} \\
\left(\Xi^{R}\right)^{T} & \Psi_{2}^{R}
\end{array}\right) \leqslant 0 \text { and }\left(\begin{array}{cc}
\Psi_{1}^{I} & \Xi^{I} \\
\left(\Xi^{I}\right)^{T} & \Psi_{2}^{I}
\end{array}\right) \leqslant 0
$$

where $\Psi_{1}^{R}=I_{N} \otimes\left(-\sum_{q=1}^{\kappa} \frac{2 A}{\xi_{q}^{2}}-2 B+\tilde{D}^{R}+2 L^{R}+\tilde{D}^{I}+\tilde{C}^{R}+\tilde{C}^{I}-\Upsilon^{R}+2 \zeta^{R} \Gamma\right)+h H \otimes\left(M+M^{T}\right), \Xi^{R}=\Xi^{I}=I_{N} \otimes\left(I_{n}-\frac{1}{2} K_{1}^{T}\right)$, $\Psi_{1}^{I}=I_{N} \otimes\left(-\sum_{q=1}^{K} \frac{2 A}{\xi_{q}^{2}}-2 B+2 L^{I}+\tilde{D}^{R}+\tilde{D}^{I}+\tilde{C}^{I}+\tilde{C}^{R}-\Upsilon^{I}+2 \zeta^{I} \Gamma\right)+h H \otimes\left(M+M^{T}\right), \Psi_{2}^{R}=\Psi_{2}^{I}=I_{N} \otimes\left(-\frac{1}{2}\left(K_{2}^{T}+K_{2}\right)-\rho I_{n}\right)$, then the network (6) is said to be passive under the controller (7).

Proof. Choose the following Lyapunov functional:

$$
\begin{aligned}
V(t)= & \sum_{s=1}^{N} \int_{\Omega}\left(e_{s}^{R}(\varrho, t)\right)^{T} e_{s}^{R}(\varrho, t) d \varrho+2 \sum_{s=1}^{N} \sum_{j=1}^{n} \int_{t-\tau_{j}(t)}^{t} \int_{\Omega} \frac{\left(\eta_{j}^{R} e_{s j}^{R}(\varrho, \delta)\right)^{2}}{1-\gamma_{j}} d \varrho d \delta \\
& +2 \sum_{s=1}^{N} \sum_{j=1}^{n} \int_{t-\tau_{j}(t)}^{t} \int_{\Omega} \frac{\left(\eta_{j}^{I} e_{s j}^{I}(\varrho, \delta)\right)^{2}}{1-\gamma_{j}} d \varrho d \delta+\sum_{s=1}^{N} \int_{\Omega}\left(e_{s}^{I}(\varrho, t)\right)^{T} e_{s}^{I}(\varrho, t) d \varrho .
\end{aligned}
$$

Then,

$$
\begin{aligned}
\dot{V}(t) \leqslant & 2 \sum_{s=1}^{N} \int_{\Omega}\left(e_{s}^{R}(\varrho, t)\right)^{T} \frac{\partial e_{s}^{R}(\varrho, t)}{\partial t} d \varrho+2 \sum_{s=1}^{N} \int_{\Omega}\left(e_{s}^{I}(\varrho, t)\right)^{T} \frac{\partial e_{s}^{I}(\varrho, t)}{\partial t} d \varrho+2 \sum_{s=1}^{N} \int_{\Omega}\left(e_{s}^{R}(\varrho, t)\right)^{T} \zeta^{R} \Gamma e_{s}^{R}(\varrho, t) d \varrho \\
& -2 \sum_{s=1}^{N} \int_{\Omega}\left(\overline{\left.e_{s}^{R}(\varrho, t)\right)^{T}} \zeta^{R} \overline{e_{s}^{R}(\varrho, t)} d \varrho+2 \sum_{s=1}^{N} \int_{\Omega}\left(e_{s}^{I}(\varrho, t)\right)^{T} \zeta^{I} \Gamma e_{s}^{I}(\varrho, t) d \varrho-2 \sum_{s=1}^{N} \int_{\Omega}\left(\overline{e_{s}^{I}(\varrho, t)}\right)^{T} \zeta^{I} \overline{e_{s}^{I}(\varrho, t)} d \varrho\right. \\
= & 2 \sum_{s=1}^{N} \int_{\Omega}\left(e_{s}^{R}(\varrho, t)\right)^{T}\left(A \triangle e_{s}^{R}(\varrho, t)-B e_{s}^{R}(\varrho, t)+D^{R}\left(Y_{s}^{R}(\varrho, t)\right) P^{R}\left(e_{s}^{R}(\varrho, t)\right)-D^{I}\left(Y_{s}^{I}(\varrho, t)\right) P^{I}\left(e_{s}^{I}(\varrho, t)\right)+x_{s}^{R}(\varrho, t)\right.
\end{aligned}
$$




$$
\begin{aligned}
& +C^{R}\left(Y_{s}^{R}(\varrho, t)\right) Q^{R}\left(\overline{e_{s}^{R}(\varrho, t)}\right)-C^{I}\left(Y_{s}^{I}(\varrho, t)\right) Q^{I}\left(\overline{e_{s}^{I}(\varrho, t)}\right)+h \sum_{k=1}^{N} H_{s k} M e_{\kappa}^{R}(\varrho, t)-\Upsilon^{R} e_{s}^{R}(\varrho, t)+\left(D^{R}\left(Y_{s}^{R}(\varrho, t)\right)\right. \\
& \left.-D^{R}\left(Y_{0}^{R}(\varrho, t)\right)\right) f^{R}\left(Y_{0}^{R}(\varrho, t)\right)-\operatorname{sign}\left(e_{s}^{R}(\varrho, t)\right)\left(\bar{D}^{R} \bar{F}^{R}+\bar{D}^{I} \bar{F}^{I}+\bar{C}^{R} \bar{G}^{R}+\bar{C}^{I} \bar{G}^{I}\right)-\left(D^{I}\left(Y_{s}^{I}(\varrho, t)\right)-D^{I}\left(Y_{0}^{I}(\varrho, t)\right)\right) \\
& \left.\times f^{I}\left(Y_{0}^{I}(\varrho, t)\right)+\left(C^{R}\left(Y_{s}^{R}(\varrho, t)\right)-C^{R}\left(Y_{0}^{R}(\varrho, t)\right)\right) g^{R}\left(Y_{0}^{R}(\varrho, t)\right)-\left(C^{I}\left(Y_{s}^{I}(\varrho, t)\right)-C^{I}\left(Y_{0}^{I}(\varrho, t)\right)\right) g^{I}\left(Y_{0}^{I}(\varrho, t)\right)\right) d \varrho \\
& +2 \sum_{s=1}^{N} \int_{\Omega}\left(e_{s}^{I}(\varrho, t)\right)^{T}\left(A \triangle e_{s}^{I}(\varrho, t)-B e_{s}^{I}(\varrho, t)+D^{R}\left(Y_{s}^{R}(\varrho, t)\right) P^{I}\left(e_{s}^{I}(\varrho, t)\right)+D^{I}\left(Y_{s}^{I}(\varrho, t)\right) P^{R}\left(e_{s}^{R}(\varrho, t)\right)+x_{s}^{I}(\varrho, t)\right. \\
& +C^{R}\left(Y_{s}^{R}(\varrho, t)\right) Q^{I}\left(\overline{e_{s}^{I}(\varrho, t)}\right)+C^{I}\left(Y_{s}^{I}(\varrho, t)\right) Q^{R}\left(\overline{e_{s}^{R}(\varrho, t)}\right)+h \sum_{k=1}^{N} H_{s \kappa} M e_{\kappa}^{I}(\varrho, t)+\left(D^{R}\left(Y_{s}^{R}(\varrho, t)\right)-D^{R}\left(Y_{0}^{R}(\varrho, t)\right)\right) \\
& \times f^{I}\left(Y_{0}^{I}(\varrho, t)\right)+\left(D^{I}\left(Y_{s}^{I}(\varrho, t)\right)-D^{I}\left(Y_{0}^{I}(\varrho, t)\right)\right) f^{R}\left(Y_{0}^{R}(\varrho, t)\right)+\left(C^{R}\left(Y_{s}^{R}(\varrho, t)\right)-C^{R}\left(Y_{0}^{R}(\varrho, t)\right)\right) g^{I}\left(Y_{0}^{I}(\varrho, t)\right) \\
& -\Upsilon^{I} e_{s}^{I}(\varrho, t)-\operatorname{sign}\left(e_{s}^{I}(\varrho, t)\right)\left(\bar{D}^{R} \bar{F}^{I}+\bar{D}^{I} \bar{F}^{R}+\bar{C}^{R} \bar{G}^{I}+\bar{C}^{I} \bar{G}^{R}\right)+\left(C^{I}\left(Y_{s}^{I}(\varrho, t)\right)-C^{I}\left(Y_{0}^{I}(\varrho, t)\right)\right) \\
& \left.\left.\times g^{R}\left(Y_{0}^{R}(\varrho, t)\right)\right) d \varrho+2 \int_{\Omega}\left(e^{R}(\varrho, t)\right)^{T}\left(I_{N} \otimes\left(\zeta^{R} \Gamma\right)\right) e^{R}(\varrho, t) d \varrho-2 \int_{\Omega} \overline{\left(e^{R}(\varrho, t)\right.}\right)^{T}\left(I_{N} \otimes \zeta^{R}\right) e^{R}(\varrho, t) d \varrho \\
& +2 \int_{\Omega}\left(e^{I}(\varrho, t)\right)^{T}\left(I_{N} \otimes\left(\zeta^{I} \Gamma\right)\right) e^{I}(\varrho, t) d \varrho-2 \int_{\Omega} \overline{\left(e^{I}(\varrho, t)\right)^{T}\left(I_{N} \otimes \zeta^{I}\right) \overline{e^{I}(\varrho, t)} d \varrho .}
\end{aligned}
$$

From the Green's formula, we have

$$
\int_{\Omega} e_{s j}^{R}(\varrho, t) \Delta e_{s j}^{R}(\varrho, t) d \varrho=-\sum_{q=1}^{K} \int_{\Omega}\left(\frac{\partial e_{s j}^{R}(\varrho, t)}{\partial \varrho_{q}}\right)^{2} d \varrho
$$

where $j=1,2, \cdots, n, s=1,2, \cdots, N$. Let $\pi^{R}(\varrho, t)=\left(I_{N} \otimes \sqrt{A}\right) e^{R}(\varrho, t)$. According to Lemma 2.1, then

$$
\begin{aligned}
& \sum_{s=1}^{N} \int_{\Omega}\left(e_{s}^{R}(\varrho, t)\right)^{T} A \triangle e_{s}^{R}(\varrho, t) d \varrho \\
= & \sum_{s=1}^{N} \sum_{j=1}^{n} a_{j} \int_{\Omega} e_{s j}^{R}(\varrho, t) \Delta e_{s j}^{R}(\varrho, t) d \varrho \\
= & -\sum_{q=1}^{K} \sum_{s=1}^{N} \sum_{j=1}^{n} a_{j} \int_{\Omega}\left(\frac{\partial e_{s j}^{R}(\varrho, t)}{\partial \varrho_{q}}\right)^{2} d \varrho \\
= & -\sum_{q=1}^{K} \int_{\Omega}\left(\frac{\partial e^{R}(\varrho, t)}{\partial \varrho_{q}}\right)^{T}\left(I_{N} \otimes A\right) \frac{\partial e^{R}(\varrho, t)}{\partial \varrho q} d \varrho \\
= & -\sum_{q=1}^{K} \int_{\Omega}\left(\frac{\partial \pi^{R}(\varrho, t)}{\partial \varrho_{q}}\right)^{T} \frac{\partial \pi^{R}(\varrho, t)}{\partial \varrho_{q}} d \varrho \\
\leqslant & -\sum_{q=1}^{K} \frac{1}{\xi_{q}^{2}} \int_{\Omega}\left(\pi^{R}(\varrho, t)\right)^{T} \pi^{R}(\varrho, t) d \varrho \\
= & -\sum_{q=1}^{K} \frac{1}{\xi_{q}^{2}} \int_{\Omega}\left(e^{R}(\varrho, t)\right)^{T}\left(I_{N} \otimes A\right) e^{R}(\varrho, t) d \varrho .
\end{aligned}
$$

Similarly,

$$
\sum_{s=1}^{N} \int_{\Omega}\left(e_{s}^{I}(\varrho, t)\right)^{T} A \triangle e_{s}^{I}(\varrho, t) d \varrho \leqslant-\sum_{q=1}^{\kappa} \frac{1}{\xi_{q}^{2}} \int_{\Omega}\left(e^{I}(\varrho, t)\right)^{T}\left(I_{N} \otimes A\right) e^{I}(\varrho, t) d \varrho .
$$


Furthermore,

$$
\begin{aligned}
& 2 \sum_{s=1}^{N} \int_{\Omega}\left(e_{s}^{R}(\varrho, t)\right)^{T} D^{R}\left(Y_{s}^{R}(\varrho, t)\right) P^{R}\left(e_{s}^{R}(\varrho, t)\right) d \varrho \\
= & 2 \sum_{s=1}^{N} \sum_{l=1}^{n} \sum_{j=1}^{n} \int_{\Omega} e_{s l}^{R}(\varrho, t) d_{\iota j}^{R}\left(Y_{s l}^{R}(\varrho, t)\right)\left(f_{j}^{R}\left(Y_{s j}^{R}(\varrho, t)\right)-f_{j}^{R}\left(Y_{0 j}^{R}(\varrho, t)\right)\right) d \varrho \\
\leqslant & 2 \sum_{s=1}^{N} \sum_{l=1}^{n} \sum_{j=1}^{n} \int_{\Omega}\left|e_{s l}^{R}(\varrho, t)\right| \tilde{d}_{\iota j}^{R}\left|f_{j}^{R}\left(Y_{s j}^{R}(\varrho, t)\right)-f_{j}^{R}\left(Y_{0 j}^{R}(\varrho, t)\right)\right| d \varrho \\
\leqslant & \sum_{s=1}^{N} \sum_{l=1}^{n} \sum_{j=1}^{n} \int_{\Omega}\left(e_{s \iota}^{R}(\varrho, t)\right)^{2}\left(\tilde{d}_{\iota j}^{R}\right)^{2} d \varrho+\sum_{s=1}^{N} \sum_{j=1}^{n} \int_{\Omega}\left(l_{j}^{R}\right)^{2}\left(e_{s j}^{R}(\varrho, t)\right)^{2} d \varrho \\
= & \int_{\Omega}\left(e^{R}(\varrho, t)\right)^{T}\left(I_{N} \otimes \tilde{D}^{R}\right) e^{R}(\varrho, t) d \varrho+\int_{\Omega}\left(e^{R}(\varrho, t)\right)^{T}\left(I_{N} \otimes L^{R}\right) e^{R}(\varrho, t) d \varrho .
\end{aligned}
$$

Similarly,

$$
\begin{aligned}
& -2 \sum_{s=1}^{N} \int_{\Omega}\left(e_{s}^{R}(\varrho, t)\right)^{T} D^{I}\left(Y_{s}^{I}(\varrho, t)\right) P^{I}\left(e_{s}^{I}(\varrho, t)\right) d \varrho \\
\leqslant & \int_{\Omega}\left(e^{R}(\varrho, t)\right)^{T}\left(I_{N} \otimes \tilde{D}^{I}\right) e^{R}(\varrho, t) d \varrho+\int_{\Omega}\left(e^{I}(\varrho, t)\right)^{T}\left(I_{N} \otimes L^{I}\right) e^{I}(\varrho, t) d \varrho \\
& 2 \sum_{s=1}^{N} \int_{\Omega}\left(e_{s}^{I}(\varrho, t)\right)^{T} D^{R}\left(Y_{s}^{R}(\varrho, t)\right) P^{I}\left(e_{s}^{I}(\varrho, t)\right) d \varrho \\
\leqslant & \int_{\Omega}\left(e^{I}(\varrho, t)\right)^{T}\left(I_{N} \otimes \tilde{D}^{R}\right) e^{I}(\varrho, t) d \varrho+\int_{\Omega}\left(e^{I}(\varrho, t)\right)^{T}\left(I_{N} \otimes L^{I}\right) e^{I}(\varrho, t) d \varrho \\
& 2 \sum_{s=1}^{N} \int_{\Omega}\left(e_{s}^{I}(\varrho, t)\right)^{T} D^{I}\left(Y_{s}^{I}(\varrho, t)\right) P^{R}\left(e_{s}^{R}(\varrho, t)\right) d \varrho \\
\leqslant & \int_{\Omega}\left(e^{I}(\varrho, t)\right)^{T}\left(I_{N} \otimes \tilde{D}^{I}\right) e^{I}(\varrho, t) d \varrho+\int_{\Omega}\left(e^{R}(\varrho, t)\right)^{T}\left(I_{N} \otimes L^{R}\right) e^{R}(\varrho, t) d \varrho .
\end{aligned}
$$

Moreover,

$$
\begin{aligned}
& 2 \sum_{s=1}^{N} \int_{\Omega}\left(e_{s}^{R}(\varrho, t)\right)^{T} C^{R}\left(Y_{s}^{R}(\varrho, t)\right) Q^{R}\left(\overline{e_{s}^{R}(\varrho, t)}\right) d \varrho \\
= & 2 \sum_{s=1}^{N} \sum_{\iota=1}^{n} \sum_{j=1}^{n} \int_{\Omega} e_{s \iota}^{R}(\varrho, t) c_{\iota j}^{R}\left(Y_{s \iota}^{R}(\varrho, t)\right)\left(g_{j}^{R}\left(Y_{s j}^{R}\left(\varrho, t-\tau_{j}(t)\right)\right)-g_{j}^{R}\left(Y_{0 j}^{R}(\varrho, t)\right)\right) d \varrho \\
\leqslant & 2 \sum_{s=1}^{N} \sum_{\iota=1}^{n} \sum_{j=1}^{n} \int_{\Omega}\left|e_{s \iota}^{R}(\varrho, t)\right| \tilde{c}_{\iota j}^{R}\left|g_{j}^{R}\left(Y_{s j}^{R}\left(\varrho, t-\tau_{j}(t)\right)\right)-g_{j}^{R}\left(Y_{0 j}^{R}(\varrho, t)\right)\right| d \varrho \\
\leqslant & \sum_{s=1}^{N} \sum_{\iota=1}^{n} \sum_{j=1}^{n} \int_{\Omega}\left(e_{s \iota}^{R}(\varrho, t)\right)^{2}\left(\tilde{c}_{\iota j}^{R}\right)^{2} d \varrho+\sum_{s=1}^{N} \sum_{j=1}^{n} \int_{\Omega}\left(\eta_{j}^{R}\right)^{2}\left(e_{s j}^{R}\left(\varrho, t-\tau_{j}(t)\right)\right)^{2} d \varrho \\
= & \left.\int_{\Omega}\left(e^{R}(\varrho, t)\right)^{T}\left(I_{N} \otimes \tilde{C}^{R}\right) e^{R}(\varrho, t) d \varrho+\int_{\Omega} \overline{\left(e^{R}(\varrho, t)\right.}\right)^{T}\left(I_{N} \otimes \zeta^{R}\right) \overline{e^{R}(\varrho, t)} d \varrho .
\end{aligned}
$$

Similarly,

$$
-2 \sum_{s=1}^{N} \int_{\Omega}\left(e_{s}^{R}(\varrho, t)\right)^{T} C^{I}\left(Y_{s}^{I}(\varrho, t)\right) Q^{I}\left(\overline{e_{s}^{I}(\varrho, t)}\right) d \varrho
$$




$$
\begin{aligned}
\leqslant & \int_{\Omega}\left(e^{R}(\varrho, t)\right)^{T}\left(I_{N} \otimes \tilde{C}^{I}\right) e^{R}(\varrho, t) d \varrho+\int_{\Omega}\left(\overline{e^{I}(\varrho, t)}\right)^{T}\left(I_{N} \otimes \zeta^{I}\right) \overline{e^{I}(\varrho, t)} d \varrho, \\
& 2 \sum_{s=1}^{N} \int_{\Omega}\left(e_{s}^{I}(\varrho, t)\right)^{T} C^{R}\left(Y_{s}^{R}(\varrho, t)\right) Q^{I}\left(\overline{e_{s}^{I}(\varrho, t)}\right) d \varrho \\
\leqslant & \int_{\Omega}\left(e^{I}(\varrho, t)\right)^{T}\left(I_{N} \otimes \tilde{C}^{R}\right) e^{I}(\varrho, t) d \varrho+\int_{\Omega}\left(\overline{e^{I}(\varrho, t)}\right)^{T}\left(I_{N} \otimes \zeta^{I}\right) \overline{e^{I}(\varrho, t)} d \varrho, \\
& 2 \sum_{s=1}^{N} \int_{\Omega}\left(e_{s}^{I}(\varrho, t)\right)^{T} C^{I}\left(Y_{s}^{I}(\varrho, t)\right) Q^{R}\left(\overline{e_{s}^{R}(\varrho, t)}\right) d \varrho \\
\leqslant & \int_{\Omega}\left(e^{I}(\varrho, t)\right)^{T}\left(I_{N} \otimes \tilde{C}^{I}\right) e^{I}(\varrho, t) d \varrho+\int_{\Omega}\left(\overline{e^{R}(\varrho, t)}\right)^{T}\left(I_{N} \otimes \zeta^{R}\right) \overline{e^{R}(\varrho, t)} d \varrho .
\end{aligned}
$$

In addition,

$$
2 h \sum_{s=1}^{N} \sum_{k=1}^{N} \int_{\Omega}\left(e_{s}^{R}(\varrho, t)\right)^{T} H_{s K} M e_{\kappa}^{R}(\varrho, t) d \varrho=h \int_{\Omega}\left(e^{R}(\varrho, t)\right)^{T}\left(H \otimes\left(M+M^{T}\right)\right) e^{R}(\varrho, t) d \varrho
$$

Similarly,

$$
2 h \sum_{s=1}^{N} \sum_{k=1}^{N} \int_{\Omega}\left(e_{s}^{I}(\varrho, t)\right)^{T} H_{s K} M e_{\kappa}^{I}(\varrho, t) d \varrho=h \int_{\Omega}\left(e^{I}(\varrho, t)\right)^{T}\left(H \otimes\left(M+M^{T}\right)\right) e^{I}(\varrho, t) d \varrho .
$$

What's more,

$$
\begin{aligned}
& 2 \sum_{s=1}^{N} \int_{\Omega}\left(e_{s}^{R}(\varrho, t)\right)^{T}\left(D^{R}\left(Y_{s}^{R}(\varrho, t)\right)-D^{R}\left(Y_{0}^{R}(\varrho, t)\right)\right) f^{R}\left(Y_{0}^{R}(\varrho, t)\right) d \varrho \\
= & 2 \sum_{s=1}^{N} \sum_{l=1}^{n} \sum_{j=1}^{n} \int_{\Omega} e_{s \iota}^{R}(\varrho, t)\left(d_{\iota j}^{R}\left(Y_{s \iota}^{R}(\varrho, t)\right)-d_{\iota j}^{R}\left(Y_{0 \iota}^{R}(\varrho, t)\right)\right) f_{j}^{R}\left(Y_{0 j}^{R}(\varrho, t)\right) d \varrho \\
\leqslant & 2 \sum_{s=1}^{N} \sum_{l=1}^{n} \sum_{j=1}^{n} \int_{\Omega}\left|e_{s \iota}^{R}(\varrho, t)\right|\left|\hat{d}_{\iota j}^{R}-\breve{d}_{\iota j}^{R}\right| F_{j}^{R} d \varrho \\
= & 2 \sum_{s=1}^{N} \int_{\Omega}\left|\left(e_{s}^{R}(\varrho, t)\right)^{T}\right| \bar{D}^{R} \bar{F}^{R} d \varrho .
\end{aligned}
$$

Similarly,

$$
\begin{aligned}
& 2 \sum_{s=1}^{N} \int_{\Omega}\left(e_{s}^{R}(\varrho, t)\right)^{T}\left(D^{I}\left(Y_{0}^{I}(\varrho, t)\right)-D^{I}\left(Y_{s}^{I}(\varrho, t)\right)\right) f^{I}\left(Y_{0}^{I}(\varrho, t)\right) d \varrho \leqslant 2 \sum_{s=1}^{N} \int_{\Omega}\left|\left(e_{s}^{R}(\varrho, t)\right)^{T}\right| \bar{D}^{I} \bar{F}^{I} d \varrho \\
& 2 \sum_{s=1}^{N} \int_{\Omega}\left(e_{s}^{I}(\varrho, t)\right)^{T}\left(D^{R}\left(Y_{s}^{R}(\varrho, t)\right)-D^{R}\left(Y_{0}^{R}(\varrho, t)\right)\right) f^{I}\left(Y_{0}^{I}(\varrho, t)\right) d \varrho \leqslant 2 \sum_{s=1}^{N} \int_{\Omega}\left|\left(e_{s}^{I}(\varrho, t)\right)^{T}\right| \bar{D}^{R} \bar{F}^{I} d \varrho, \\
& 2 \sum_{s=1}^{N} \int_{\Omega}\left(e_{s}^{I}(\varrho, t)\right)^{T}\left(D^{I}\left(Y_{s}^{I}(\varrho, t)\right)-D^{I}\left(Y_{0}^{I}(\varrho, t)\right)\right) f^{R}\left(Y_{0}^{R}(\varrho, t)\right) d \varrho \leqslant 2 \sum_{s=1}^{N} \int_{\Omega}\left|\left(e_{s}^{I}(\varrho, t)\right)^{T}\right| \bar{D}^{I} \bar{F}^{R} d \varrho
\end{aligned}
$$

In addition, we have

$$
2 \sum_{s=1}^{N} \int_{\Omega}\left(e_{s}^{R}(\varrho, t)\right)^{T}\left(C^{R}\left(Y_{s}^{R}(\varrho, t)\right)-C^{R}\left(Y_{0}^{R}(\varrho, t)\right)\right) g^{R}\left(Y_{0}^{R}(\varrho, t)\right) d \varrho
$$




$$
\begin{aligned}
& =2 \sum_{s=1}^{N} \sum_{l=1}^{n} \sum_{j=1}^{n} \int_{\Omega} e_{s \iota}^{R}(\varrho, t)\left(c_{\iota j}^{R}\left(Y_{s \iota}^{R}(\varrho, t)\right)-c_{\iota j}^{R}\left(Y_{0 \iota}^{R}(\varrho, t)\right)\right) g_{j}^{R}\left(Y_{0 j}^{R}(\varrho, t)\right) d \varrho \\
& \leqslant 2 \sum_{s=1}^{N} \sum_{\iota=1}^{n} \sum_{j=1}^{n} \int_{\Omega}\left|e_{s \iota}^{R}(\varrho, t) \| \hat{c}_{\iota j}^{R}-\check{c}_{\iota j}^{R}\right| G_{j}^{R} d \varrho \\
& =2 \sum_{s=1}^{N} \int_{\Omega}\left|\left(e_{s}^{R}(\varrho, t)\right)^{T}\right| \bar{C}^{R} \bar{G}^{R} d \varrho .
\end{aligned}
$$

Similarly,

$$
\begin{aligned}
& 2 \sum_{s=1}^{N} \int_{\Omega}\left(e_{s}^{R}(\varrho, t)\right)^{T}\left(C^{I}\left(Y_{0}^{I}(\varrho, t)\right)-C^{I}\left(Y_{s}^{I}(\varrho, t)\right)\right) g^{I}\left(Y_{0}^{I}(\varrho, t)\right) d \varrho \leqslant 2 \sum_{s=1}^{N} \int_{\Omega}\left|\left(e_{s}^{R}(\varrho, t)\right)^{T}\right| \bar{C}^{I} \bar{G}^{I} d \varrho \\
& 2 \sum_{s=1}^{N} \int_{\Omega}\left(e_{s}^{I}(\varrho, t)\right)^{T}\left(C^{R}\left(Y_{s}^{R}(\varrho, t)\right)-C^{R}\left(Y_{0}^{R}(\varrho, t)\right)\right) g^{I}\left(Y_{0}^{I}(\varrho, t)\right) d \varrho \leqslant 2 \sum_{s=1}^{N} \int_{\Omega}\left|\left(e_{s}^{I}(\varrho, t)\right)^{T}\right| \bar{C}^{R} \bar{G}^{I} d \varrho, \\
& 2 \sum_{s=1}^{N} \int_{\Omega}\left(e_{s}^{I}(\varrho, t)\right)^{T}\left(C^{I}\left(Y_{s}^{I}(\varrho, t)\right)-C^{I}\left(Y_{0}^{I}(\varrho, t)\right)\right) g^{R}\left(Y_{0}^{R}(\varrho, t)\right) d \varrho \leqslant 2 \sum_{s=1}^{N} \int_{\Omega}\left|\left(e_{s}^{I}(\varrho, t)\right)^{T}\right| \bar{C}^{I} \bar{G}^{R} d \varrho .
\end{aligned}
$$

From (9) to (29), we have

$$
\begin{aligned}
\dot{V}(t) \leqslant & \int_{\Omega}\left(e^{R}(\varrho, t)\right)^{T}\left(I_{N} \otimes\left(-\sum_{q=1}^{K} \frac{2 A}{\xi_{q}^{2}}-2 B+\tilde{D}^{R}+2 L^{R}+\tilde{D}^{I}+\tilde{C}^{R}+\tilde{C}^{I}-\Upsilon^{R}+2 \zeta^{R} \Gamma\right)+h H \otimes\left(M+M^{T}\right)\right) e^{R}(\varrho, t) d \varrho \\
& +\int_{\Omega}\left(e^{I}(\varrho, t)\right)^{T}\left(I_{N} \otimes\left(-\sum_{q=1}^{K} \frac{2 A}{\xi_{q}^{2}}-2 B+2 L^{I}+\tilde{D}^{R}+\tilde{D}^{I}+\tilde{C}^{I}+\tilde{C}^{R}-\Upsilon^{I}+2 \zeta^{I} \Gamma\right)+h H \otimes\left(M+M^{T}\right)\right) \\
& \times e^{I}(\varrho, t) d \varrho+2 \int_{\Omega}\left(e^{R}(\varrho, t)\right)^{T} x^{R}(\varrho, t) d \varrho+2 \int_{\Omega}\left(e^{I}(\varrho, t)\right)^{T} x^{I}(\varrho, t) d \varrho .
\end{aligned}
$$

Furthermore,

$$
\begin{aligned}
& \dot{V}(t)-\int_{\Omega}\left[\left(z^{R}(\varrho, t)\right)^{T} x^{R}(\varrho, t)+\left(z^{I}(\varrho, t)\right)^{T} x^{I}(\varrho, t)\right] d \varrho-\rho \int_{\Omega}\left[\left(x^{R}(\varrho, t)\right)^{T} x^{R}(\varrho, t)+\left(x^{I}(\varrho, t)\right)^{T} x^{I}(\varrho, t)\right] d \varrho \\
& \leqslant \int_{\Omega}\left(e^{R}(\varrho, t)\right)^{T}\left(I_{N} \otimes\left(-\sum_{q=1}^{K} \frac{2 A}{\xi_{q}^{2}}-2 B+\tilde{D}^{R}+2 L^{R}+\tilde{D}^{I}+\tilde{C}^{R}+\tilde{C}^{I}-\Upsilon^{R}+2 \zeta^{R} \Gamma\right)+h H \otimes(M\right. \\
& \left.\left.+M^{T}\right)\right) e^{R}(\varrho, t) d \varrho+\int_{\Omega}\left(e^{I}(\varrho, t)\right)^{T}\left(I _ { N } \otimes \left(-\sum_{q=1}^{K} \frac{2 A}{\xi_{q}^{2}}-2 B+2 L^{I}+\widetilde{D}^{R}+\tilde{D}^{I}+\tilde{C}^{I}+\tilde{C}^{R}-\Upsilon^{I}\right.\right. \\
& \left.\left.+2 \zeta^{I} \Gamma\right)+h H \otimes\left(M+M^{T}\right)\right) e^{I}(\varrho, t) d \varrho+\int_{\Omega}\left(x^{R}(\varrho, t)\right)^{T}\left(I_{N} \otimes\left(-\frac{1}{2}\left(K_{2}^{T}+K_{2}\right)-\rho I_{n}\right)\right) x^{R}(\varrho, t) d \varrho \\
& +2 \int_{\Omega}\left(e^{R}(\varrho, t)\right)^{T}\left(I_{N} \otimes\left(I_{n}-\frac{1}{2} K_{1}^{T}\right)\right) x^{R}(\varrho, t) d \varrho+\int_{\Omega}\left(x^{I}(\varrho, t)\right)^{T}\left(I_{N} \otimes\left(-\frac{1}{2}\left(K_{2}^{T}+K_{2}\right)-\rho I_{n}\right)\right) x^{I}(\varrho, t) d \varrho \\
& +2 \int_{\Omega}\left(e^{I}(\varrho, t)\right)^{T}\left(I_{N} \otimes\left(I_{n}-\frac{1}{2} K_{1}^{T}\right)\right) x^{I}(\varrho, t) d \varrho \\
& =\int_{\Omega}\left(\varphi^{R}(\varrho, t)\right)^{T}\left(\begin{array}{cc}
\Psi_{1}^{R} & \Xi^{R} \\
\left(\Xi^{R}\right)^{T} & \Psi_{2}^{R}
\end{array}\right) \varphi^{R}(\varrho, t) d \varrho+\int_{\Omega}\left(\varphi^{I}(\varrho, t)\right)^{T}\left(\begin{array}{cc}
\Psi_{1}^{I} & \Xi^{I} \\
\left(\Xi^{I}\right)^{T} & \Psi_{2}^{I}
\end{array}\right) \varphi^{I}(\varrho, t) d \varrho,
\end{aligned}
$$

where $\varphi^{R}(\varrho, t)=\left(\left(e^{R}(\varrho, t)\right)^{T},\left(x^{R}(\varrho, t)\right)^{T}\right)^{T}$ and $\varphi^{I}(\varrho, t)=\left(\left(e^{I}(\varrho, t)\right)^{T},\left(x^{I}(\varrho, t)\right)^{T}\right)^{T}$. From (8), we can easily obtain

$$
\dot{V}(t) \leqslant \int_{\Omega}\left[\left(z^{R}(\varrho, t)\right)^{T} x^{R}(\varrho, t)+\left(z^{I}(\varrho, t)\right)^{T} x^{I}(\varrho, t)\right] d \varrho+\rho \int_{\Omega}\left[\left(x^{R}(\varrho, t)\right)^{T} x^{R}(\varrho, t)+\left(x^{I}(\varrho, t)\right)^{T} x^{I}(\varrho, t)\right] d \varrho .
$$


By integrating (30) about $t$ over the time period from $t_{1}$ to $t_{2}$, one has

$$
\begin{aligned}
V\left(t_{2}\right)-V\left(t_{1}\right) \leqslant & \int_{t_{1}}^{t_{2}} \int_{\Omega}\left[\left(z^{R}(\varrho, t)\right)^{T} x^{R}(\varrho, t)+\left(z^{I}(\varrho, t)\right)^{T} x^{I}(\varrho, t)\right] d \varrho d t \\
& +\rho \int_{t_{1}}^{t_{2}} \int_{\Omega}\left[\left(x^{R}(\varrho, t)\right)^{T} x^{R}(\varrho, t)+\left(x^{I}(\varrho, t)\right)^{T} x^{I}(\varrho, t)\right] d \varrho d t, t_{2} \geqslant t_{1} .
\end{aligned}
$$

Namely,

$$
\int_{t_{1}}^{t_{2}} \int_{\Omega}\left[\left(z^{R}(\varrho, t)\right)^{T} x^{R}(\varrho, t)+\left(z^{I}(\varrho, t)\right)^{T} x^{I}(\varrho, t)\right] d \varrho d t \geqslant V\left(t_{2}\right)-V\left(t_{1}\right)-\rho \int_{t_{1}}^{t_{2}} \int_{\Omega}\left[\left(x^{R}(\varrho, t)\right)^{T} x^{R}(\varrho, t)+\left(x^{I}(\varrho, t)\right)^{T} x^{I}(\varrho, t)\right] d \varrho d t
$$

for any $t_{2}, t_{1} \in \mathbb{R}^{+}$and $t_{2} \geqslant t_{1}$.

According to Definition 3.1, we can obtain that the network (6) is passive under the controller (7).

\subsection{Synchronization control}

Theorem 3.2. The network (2) is said to be synchronized under the controller (7) if

$$
\Psi_{1}^{R}<0 \text { and } \Psi_{1}^{I}<0
$$

where $\Psi_{1}^{R}=I_{N} \otimes\left(-\sum_{q=1}^{k} \frac{2 A}{\xi_{q}^{2}}-2 B+\tilde{D}^{R}+2 L^{R}+\tilde{D}^{I}+\tilde{C}^{R}+\tilde{C}^{I}-\Upsilon^{R}+2 \zeta^{R} \Gamma\right)+h H \otimes\left(M+M^{T}\right), \Psi_{1}^{I}=I_{N} \otimes\left(-\sum_{q=1}^{k} \frac{2 A}{\xi_{q}^{2}}-\right.$ $\left.2 B+2 L^{I}+\tilde{D}^{R}+\tilde{D}^{I}+\tilde{C}^{I}+\tilde{C}^{R}-\Upsilon^{I}+2 \zeta^{I} \Gamma\right)+h H \otimes\left(M+M^{T}\right)$.

Proof. The same Lyapunov functional in this subsection is defined as in Theorem 3.1. Then, one has

$$
\begin{aligned}
\dot{V}(t) \leqslant & \int_{\Omega}\left(e^{R}(\varrho, t)\right)^{T}\left(I_{N} \otimes\left(-\sum_{q=1}^{\kappa} \frac{2 A}{\xi_{q}^{2}}-2 B+\tilde{D}^{R}+2 L^{R}+\tilde{D}^{I}+\tilde{C}^{R}+\tilde{C}^{I}-\Upsilon^{R}+2 \zeta^{R} \Gamma\right)+h H \otimes\left(M+M^{T}\right)\right) e^{R}(\varrho, t) d \varrho \\
& +\int_{\Omega}\left(e^{I}(\varrho, t)\right)^{T}\left(I_{N} \otimes\left(-\sum_{q=1}^{\kappa} \frac{2 A}{\xi_{q}^{2}}-2 B+2 L^{I}+\tilde{D}^{R}+\tilde{D}^{I}+\tilde{C}^{I}+\tilde{C}^{R}-\Upsilon^{I}+2 \zeta^{I} \Gamma\right)+h H \otimes\left(M+M^{T}\right)\right) e^{I}(\varrho, t) d \varrho \\
\leqslant & \alpha\|e(\cdot, t)\|^{2}
\end{aligned}
$$

where $\alpha=\max \left\{\lambda_{M}\left(\Psi_{1}^{R}\right), \lambda_{M}\left(\Psi_{1}^{I}\right)\right\}$.

According to (32) and the definition of $V(t)$, we can obtain $V(t)$ is non-increasing and bounded. Therefore, $\lim _{t \rightarrow+\infty} V(t)$ exists and satisfies $\lim _{t \rightarrow+\infty} V(t) \geqslant 0$. Moreover, from (32), we can get

$$
\|e(\cdot, t)\|^{2} \leqslant \frac{\dot{V}(t)}{\alpha}
$$

From (33), we can easily derive that $\lim _{t \rightarrow+\infty} \int_{0}^{t}\|e(\cdot, \delta)\|^{2} d \delta$ exists and is nonnegative real number. Moreover,

$$
\begin{aligned}
0 & \leqslant \lim _{t \rightarrow+\infty} \sum_{s=1}^{N} \sum_{j=1}^{n} \int_{t-\tau_{j}(t)}^{t} \int_{\Omega} \frac{2\left(\eta_{j}^{R} e_{s j}^{R}(\varrho, \delta)\right)^{2}}{1-\gamma_{j}} d \varrho d \delta \\
& \leqslant \lim _{t \rightarrow+\infty} \sum_{s=1}^{N} \sum_{j=1}^{n} \int_{t-\tau}^{t} \int_{\Omega} \frac{2\left(\eta_{j}^{R} e_{s j}^{R}(\varrho, \delta)\right)^{2}}{1-\gamma_{j}} d \varrho d \delta \\
& =\lim _{t \rightarrow+\infty} \int_{t-\tau}^{t} \int_{\Omega}\left(e^{R}(\varrho, \delta)\right)^{T}\left(I_{N} \otimes\left(2 \zeta^{R} \Gamma\right)\right) e^{R}(\varrho, \delta) d \varrho d \delta \\
& \leqslant \lambda_{M}\left(I_{N} \otimes\left(2 \zeta^{R} \Gamma\right)\right) \lim _{t \rightarrow+\infty} \int_{t-\tau}^{t}\left\|e^{R}(\cdot, \delta)\right\|^{2} d \delta \\
& =0 .
\end{aligned}
$$


Similarly,

$$
0 \leqslant \lim _{t \rightarrow+\infty} \sum_{s=1}^{N} \sum_{j=1}^{n} \int_{t-\tau_{j}(t)}^{t} \int_{\Omega} \frac{2\left(\eta_{j}^{I} e_{s j}^{I}(\varrho, \delta)\right)^{2}}{1-\gamma_{j}} d \varrho d \delta=0 .
$$

From (34) and (35), it is easy to know that $\lim _{t \rightarrow+\infty} \sum_{s=1}^{N} \int_{\Omega}\left[\left(e_{s}^{R}(\varrho, t)\right)^{T} e_{s}^{R}(\varrho, t)+\left(e_{s}^{I}(\varrho, t)\right)^{T} e_{s}^{I}(\varrho, t)\right] d \varrho$ exists and is a nonnegative real number. Suppose that

$$
\lim _{t \rightarrow+\infty} \sum_{s=1}^{N} \int_{\Omega}\left[\left(e_{s}^{R}(\varrho, t)\right)^{T} e_{s}^{R}(\varrho, t)+\left(e_{s}^{I}(\varrho, t)\right)^{T} e_{s}^{I}(\varrho, t)\right] d \varrho=\beta>0 .
$$

Then, there exists a real number $\epsilon>0$ satisfying

$$
\sum_{s=1}^{N} \int_{\Omega}\left[\left(e_{s}^{R}(\varrho, t)\right)^{T} e_{s}^{R}(\varrho, t)+\left(e_{s}^{I}(\varrho, t)\right)^{T} e_{s}^{I}(\varrho, t)\right] d \varrho>\frac{\beta}{2} \text { for } t \geqslant \epsilon
$$

Then, one has

$$
\|e(\cdot, t)\|^{2}>\frac{\beta}{2}, t \geqslant \epsilon
$$

Combined (32) with (36), we have

$$
\dot{V}(t)<\frac{\alpha \beta}{2}, t \geqslant \epsilon
$$

By (37), we can acquire

$$
-V(\epsilon) \leqslant V(+\infty)-V(\epsilon)=\int_{\epsilon}^{+\infty} \dot{V}(t) d t<\int_{\epsilon}^{+\infty} \frac{\alpha \beta}{2} d t=-\infty,
$$

which is unreasonable. Therefore,

$$
\lim _{t \rightarrow+\infty} \sum_{s=1}^{N} \int_{\Omega}\left[\left(e_{s}^{R}(\varrho, t)\right)^{T} e_{s}^{R}(\varrho, t)+\left(e_{s}^{I}(\varrho, t)\right)^{T} e_{s}^{I}(\varrho, t)\right] d \varrho=0 .
$$

Then, we can obtain

$$
\lim _{t \rightarrow+\infty}\|e(\cdot, t)\|=0 .
$$

Consequently, the network (2) achieves synchronization.

Remark 1. In recent years, the study of CMNNs has become a hotspot and a large number of interesting results on CMNNs have been reported [23, 25, 24, 26, 27, 28, 29]. However, the CMNN models considered in these existing works $[23,25,24,26,27,28,29]$ are real-valued. In fact, complex variables exist in many applications such as pattern recognition, associative memory, nonlinear filtering and image reconstruction. Compared with real-valued neural networks, CVNNs have more complex characteristics and more extensive practical applications. For example, the XOR and symmetry detection problems that cannot be solved by real-valued neural networks but can be easily solved by CVNNs [32]. Additionally, the models of CVNNs are more general, because the real-valued neural networks are only some special cases of them when there are no imaginary parts of complex variables. Thus, it is of great significance to study CVNNs. Moreover, CVMNNs can be built by replacing resistors with memristor in VLSI circuits of CVNNs as described in [34], which have a variety of different applications in image processing, engineering optimization, pattern recognition, etc. In addition, it is also necessary to take the reaction-diffusion term into consideration when studying CVMNNs. In more details, the CRDCVMNNs are described by complex-valued partial differential equation with memristive characteristics. Unfortunately, there is no work reported on passivity and synchronization of CRDCVMNNs. To the best of our knowledge, this paper is the first step towards investigating the dynamical behaviors of CRDCVMNNs. 


\section{Passivity and synchronization of CRDCVMNNs with spatial diffusion coupling}

\subsection{Network model}

A CRDCVMNN with spatial diffusion coupling in this section is shown as follows:

$$
\begin{aligned}
\frac{\partial Y_{s}(\varrho, t)}{\partial t}= & A \triangle Y_{s}(\varrho, t)-B Y_{s}(\varrho, t)+C\left(Y_{s}(\varrho, t)\right) g\left(\overline{Y_{s}(\varrho, t)}\right)+u_{s}(\varrho, t)+D\left(Y_{s}(\varrho, t)\right) f\left(Y_{s}(\varrho, t)\right) \\
& +\hat{h} \sum_{k=1}^{N} \hat{H}_{s k} \hat{M} \Delta Y_{\kappa}(\varrho, t)+x_{s}(\varrho, t), \quad s=1,2, \cdots, N,
\end{aligned}
$$

where $Y_{s}(\varrho, t), A, \Delta, B, C(\cdot), g(\cdot), u_{s}(\varrho, t), D(\cdot), f(\cdot), x_{s}(\varrho, t)$ are defined in the same way as those in Section 3. $\hat{h}, \hat{H}_{s K}, \hat{M}$ have the similar definitions as $h, H_{s K}, M$ in Section 3.

For network (39),

$$
\begin{aligned}
& Y_{s}(\varrho, t)=\varphi_{s}(\varrho, t) \in \mathbb{C}^{n}, \quad(\varrho, t) \in \Omega \times[-\tau, 0], \\
& Y_{s}(\varrho, t)=0, \quad(\varrho, t) \in \partial \Omega \times[-\tau,+\infty),
\end{aligned}
$$

where $\varphi_{s}(\varrho, t)=\left(\varphi_{s 1}(\varrho, t), \varphi_{s 2}(\varrho, t), \cdots, \varphi_{s n}(\varrho, t)\right)^{T} \in \mathbb{C}^{n}$ is bounded and continuous on $\Omega \times[-\tau, 0]$.

Then, the network (39) can be separated into real and imaginary parts as follows:

$$
\begin{aligned}
\frac{\partial Y_{s}^{R}(\varrho, t)}{\partial t}= & \left.A \triangle Y_{s}^{R}(\varrho, t)-B Y_{s}^{R}(\varrho, t)+C^{R}\left(Y_{s}^{R}(\varrho, t)\right) g^{R} \overline{Y_{s}^{R}(\varrho, t)}\right)+u_{s}^{R}(\varrho, t)-C^{I}\left(Y_{s}^{I}(\varrho, t)\right) g^{I}\left(\overline{Y_{s}^{I}(\varrho, t)}\right)+D^{R}\left(Y_{s}^{R}(\varrho, t)\right) f^{R}\left(Y_{s}^{R}(\varrho, t)\right) \\
& +x_{s}^{R}(\varrho, t)-D^{I}\left(Y_{s}^{I}(\varrho, t)\right) f^{I}\left(Y_{s}^{I}(\varrho, t)\right)+\hat{h} \sum_{k=1}^{N} \hat{H}_{s k} \hat{M} \Delta Y_{\kappa}^{R}(\varrho, t) \\
\frac{\partial Y_{s}^{I}(\varrho, t)}{\partial t}= & \left.A \Delta Y_{s}^{I}(\varrho, t)-B Y_{s}^{I}(\varrho, t)+C^{R}\left(Y_{s}^{R}(\varrho, t)\right) g^{I} \overline{\left(Y_{s}^{I}(\varrho, t)\right)}+u_{s}^{I}(\varrho, t)+C^{I}\left(Y_{s}^{I}(\varrho, t)\right) g^{R} \overline{Y_{s}^{R}(\varrho, t)}\right)+D^{R}\left(Y_{s}^{R}(\varrho, t)\right) f^{I}\left(Y_{s}^{I}(\varrho, t)\right) \\
& +x_{s}^{I}(\varrho, t)+D^{I}\left(Y_{s}^{I}(\varrho, t)\right) f^{R}\left(Y_{s}^{R}(\varrho, t)\right)+\hat{h} \sum_{k=1}^{N} \hat{H}_{s k} \hat{M} \Delta Y_{k}^{I}(\varrho, t) .
\end{aligned}
$$

For network (40),

$$
\begin{aligned}
& Y_{s}^{R}(\varrho, t)=\varphi_{s}^{R}(\varrho, t) \in \mathbb{R}^{n}, \quad(\varrho, t) \in \Omega \times[-\tau, 0], \\
& Y_{s}^{R}(\varrho, t)=0, \quad(\varrho, t) \in \partial \Omega \times[-\tau,+\infty),
\end{aligned}
$$

where $\varphi_{s}^{R}(\varrho, t)=\left(\varphi_{s 1}^{R}(\varrho, t), \varphi_{s 2}^{R}(\varrho, t), \cdots, \varphi_{s n}^{R}(\varrho, t)\right)^{T} \in \mathbb{R}^{n}$ is bounded and continuous on $\Omega \times[-\tau, 0]$.

For network (41),

$$
\begin{aligned}
& Y_{s}^{I}(\varrho, t)=\varphi_{s}^{I}(\varrho, t) \in \mathbb{R}^{n}, \quad(\varrho, t) \in \Omega \times[-\tau, 0], \\
& Y_{s}^{I}(\varrho, t)=0, \quad(\varrho, t) \in \partial \Omega \times[-\tau,+\infty),
\end{aligned}
$$

where $\varphi_{s}^{I}(\varrho, t)=\left(\varphi_{s 1}^{I}(\varrho, t), \varphi_{s 2}^{I}(\varrho, t), \cdots, \varphi_{s n}^{I}(\varrho, t)\right)^{T} \in \mathbb{R}^{n}$ is bounded and continuous on $\Omega \times[-\tau, 0]$.

Let $e_{s}(\varrho, t)=Y_{s}(\varrho, t)-Y_{0}(\varrho, t)$, then

$$
\begin{aligned}
\frac{\partial e_{s}(\varrho, t)}{\partial t}= & A \Delta e_{s}(\varrho, t)-B e_{s}(\varrho, t)+C\left(Y_{s}(\varrho, t)\right) g\left(\overline{Y_{s}(\varrho, t)}\right)+u_{s}(\varrho, t)-C\left(Y_{0}(\varrho, t)\right) g\left(Y_{0}(\varrho, t)\right)+D\left(Y_{s}(\varrho, t)\right) f\left(Y_{s}(\varrho, t)\right) \\
& +x_{s}(\varrho, t)-D\left(Y_{0}(\varrho, t)\right) f\left(Y_{0}(\varrho, t)\right)+\hat{h} \sum_{k=1}^{N} \hat{H}_{s \kappa} \hat{M} \triangle e_{\kappa}(\varrho, t)
\end{aligned}
$$

where $s=1,2, \cdots, N$ and $e_{s}(\varrho, t)=\left(e_{s 1}(\varrho, t), e_{s 2}(\varrho, t), \cdots, e_{s n}(\varrho, t)\right)^{T}$. 
By separating (42) into real and imaginary parts, we have

$$
\begin{aligned}
\frac{\partial e_{s}^{R}(\varrho, t)}{\partial t}= & A \triangle e_{s}^{R}(\varrho, t)-B e_{s}^{R}(\varrho, t)+D^{R}\left(Y_{s}^{R}(\varrho, t)\right) P^{R}\left(e_{s}^{R}(\varrho, t)\right)+u_{s}^{R}(\varrho, t)-D^{I}\left(Y_{s}^{I}(\varrho, t)\right) P^{I}\left(e_{s}^{I}(\varrho, t)\right)+C^{R}\left(Y_{s}^{R}(\varrho, t)\right) Q^{R}\left(\overline{e_{s}^{R}(\varrho, t)}\right) \\
& +x_{s}^{R}(\varrho, t)-C^{I}\left(Y_{s}^{I}(\varrho, t)\right) Q^{I}\left(\overline{e_{s}^{I}(\varrho, t)}\right)+\hat{h} \sum_{k=1}^{N} \hat{H}_{s k} \hat{M} \triangle e_{\kappa}^{R}(\varrho, t)+\left(D^{R}\left(Y_{s}^{R}(\varrho, t)\right)-D^{R}\left(Y_{0}^{R}(\varrho, t)\right)\right) f^{R}\left(Y_{0}^{R}(\varrho, t)\right) \\
& -\left(D^{I}\left(Y_{s}^{I}(\varrho, t)\right)-D^{I}\left(Y_{0}^{I}(\varrho, t)\right)\right) f^{I}\left(Y_{0}^{I}(\varrho, t)\right)+\left(C^{R}\left(Y_{s}^{R}(\varrho, t)\right)-C^{R}\left(Y_{0}^{R}(\varrho, t)\right)\right) g^{R}\left(Y_{0}^{R}(\varrho, t)\right) \\
& -\left(C^{I}\left(Y_{s}^{I}(\varrho, t)\right)-C^{I}\left(Y_{0}^{I}(\varrho, t)\right)\right) g^{I}\left(Y_{0}^{I}(\varrho, t)\right) \\
\frac{\partial e_{s}^{I}(\varrho, t)}{\partial t}= & A \triangle e_{s}^{I}(\varrho, t)-B e_{s}^{I}(\varrho, t)+D^{R}\left(Y_{s}^{R}(\varrho, t)\right) P^{I}\left(e_{s}^{I}(\varrho, t)\right)+u_{s}^{I}(\varrho, t)+D^{I}\left(Y_{s}^{I}(\varrho, t)\right) P^{R}\left(e_{s}^{R}(\varrho, t)\right)+C^{R}\left(Y_{s}^{R}(\varrho, t)\right) Q^{I}\left(\overline{\left.e_{s}^{I}(\varrho, t)\right)}\right. \\
& +x_{s}^{I}(\varrho, t)+C^{I}\left(Y_{s}^{I}(\varrho, t)\right) Q^{R}\left(\overline{e_{s}^{R}(\varrho, t)}\right)+\hat{h} \sum_{k=1}^{N} \hat{H}_{s \kappa} \hat{M} \triangle e_{\kappa}^{I}(\varrho, t)+\left(D^{R}\left(Y_{s}^{R}(\varrho, t)\right)-D^{R}\left(Y_{0}^{R}(\varrho, t)\right)\right) f^{I}\left(Y_{0}^{I}(\varrho, t)\right) \\
& +\left(D^{I}\left(Y_{s}^{I}(\varrho, t)\right)-D^{I}\left(Y_{0}^{I}(\varrho, t)\right)\right) f^{R}\left(Y_{0}^{R}(\varrho, t)\right)+\left(C^{R}\left(Y_{s}^{R}(\varrho, t)\right)-C^{R}\left(Y_{0}^{R}(\varrho, t)\right)\right) g^{I}\left(Y_{0}^{I}(\varrho, t)\right) \\
& +\left(C^{I}\left(Y_{s}^{I}(\varrho, t)\right)-C^{I}\left(Y_{0}^{I}(\varrho, t)\right)\right) g^{R}\left(Y_{0}^{R}(\varrho, t)\right) .
\end{aligned}
$$

Remark 2. In Section 3, we established passivity and synchronization criteria for CRDCVMNNs with state coupling. As is known, different diffusion of node may affect the states of surrounding nodes in some realistic reaction-diffusion networks. Therefore, it is very interesting to consider spatial diffusion coupling when modeling CRDCVMNNs. Moreover, CRDCVMNNs with spatial diffusion couplings can reflect more realistically characteristics of some complex networks to some extent. Therefore, we further study the dynamical behaviors of spatial diffusion CRDCVMNNs in this section, which is also one of contributions of our work.

\subsection{Passivity control}

The output vector $z_{s}(\varrho, t) \in \mathbb{C}^{n}$ of the system (42) is described as follows:

$$
z_{s}(\varrho, t)=\hat{K}_{1} e_{s}(\varrho, t)+\hat{K}_{2} x_{s}(\varrho, t),
$$

where $\hat{K}_{1} \in \mathbb{R}^{n \times n}$ and $\hat{K}_{2} \in \mathbb{R}^{n \times n}$. Moreover, the controller designed for the network (39) in this section is the same as in Section 3.

Theorem 4.1. If there exists a constant $\rho>0$ such that

$$
\begin{gathered}
2 I_{N} \otimes A+\hat{h} \hat{H} \otimes\left(\hat{M}+\hat{M}^{T}\right) \geqslant 0, \\
\left(\begin{array}{cc}
\hat{\Psi}_{1}^{R} & \hat{\Xi}^{R} \\
\left(\hat{\Xi}^{R}\right)^{T} & \hat{\Psi}_{2}^{R}
\end{array}\right) \leqslant 0 \text { and }\left(\begin{array}{cc}
\hat{\Psi}_{1}^{I} & \hat{\Xi}^{I} \\
\left(\hat{\Xi}^{I}\right)^{T} & \hat{\Psi}_{2}^{I}
\end{array}\right) \leqslant 0,
\end{gathered}
$$

where $\hat{\Psi}_{1}^{R}=I_{N} \otimes\left(-\sum_{q=1}^{\kappa} \frac{2 A}{\xi_{q}^{2}}-2 B+\tilde{D}^{R}+2 L^{R}+\tilde{D}^{I}+\tilde{C}^{R}+\tilde{C}^{I}-\Upsilon^{R}+2 \zeta^{R} \Gamma\right)-\sum_{q=1}^{K} \frac{\hat{h}}{\xi_{q}^{2}} \hat{H} \otimes\left(\hat{M}+\hat{M}^{T}\right), \hat{\Xi}^{R}=\hat{\Xi}^{I}=$ $I_{N} \otimes\left(I_{n}-\frac{1}{2} \hat{K}_{1}^{T}\right), \hat{\Psi}_{1}^{I}=I_{N} \otimes\left(-\sum_{q=1}^{\kappa} \frac{2 A}{\xi_{q}^{2}}-2 B+2 L^{I}+\tilde{D}^{R}+\tilde{D}^{I}+\tilde{C}^{I}+\tilde{C}^{R}-\Upsilon^{I}+2 \zeta^{I} \Gamma\right)-\sum_{q=1}^{k} \frac{\hat{h}}{\xi_{q}^{2}} \hat{H} \otimes\left(\hat{M}+\hat{M}^{T}\right)$, $\hat{\Psi}_{2}^{R}=\hat{\Psi}_{2}^{I}=I_{N} \otimes\left(-\frac{1}{2}\left(\hat{K}_{2}^{T}+\hat{K}_{2}\right)-\rho I_{n}\right)$, then the network (42) is said to be passive under the controller (7).

Proof. Choose the same Lyapunov functional $V(t)$ for network (42) as in Theorem 3.1, then we have

$$
\begin{aligned}
\dot{V}(t) \leqslant & 2 \sum_{s=1}^{N} \int_{\Omega}\left(e_{s}^{R}(\varrho, t)\right)^{T}\left(A \triangle e_{s}^{R}(\varrho, t)-B e_{s}^{R}(\varrho, t)+D^{R}\left(Y_{s}^{R}(\varrho, t)\right) P^{R}\left(e_{s}^{R}(\varrho, t)\right)-D^{I}\left(Y_{s}^{I}(\varrho, t)\right) P^{I}\left(e_{s}^{I}(\varrho, t)\right)\right. \\
& +C^{R}\left(Y_{s}^{R}(\varrho, t)\right) Q^{R}\left(\overline{e_{s}^{R}(\varrho, t)}\right)+x_{s}^{R}(\varrho, t)-C^{I}\left(Y_{s}^{I}(\varrho, t)\right) Q^{I}\left(\overline{e_{s}^{I}(\varrho, t)}\right)+\hat{h} \sum_{\kappa=1}^{N} \hat{H}_{s \kappa} \hat{M} \triangle e_{\kappa}^{R}(\varrho, t)
\end{aligned}
$$




$$
\begin{aligned}
& +\left(D^{R}\left(Y_{s}^{R}(\varrho, t)\right)-D^{R}\left(Y_{0}^{R}(\varrho, t)\right)\right) f^{R}\left(Y_{0}^{R}(\varrho, t)\right)-\Upsilon^{R} e_{s}^{R}(\varrho, t)-\operatorname{sign}\left(e_{s}^{R}(\varrho, t)\right)\left(\bar{D}^{R} \bar{F}^{R}\right. \\
& \left.+\bar{D}^{I} \bar{F}^{I}+\bar{C}^{R} \bar{G}^{R}+\bar{C}^{I} \bar{G}^{I}\right)-\left(D^{I}\left(Y_{s}^{I}(\varrho, t)\right)-D^{I}\left(Y_{0}^{I}(\varrho, t)\right)\right) f^{I}\left(Y_{0}^{I}(\varrho, t)\right) \\
& +\left(C^{R}\left(Y_{s}^{R}(\varrho, t)\right)-C^{R}\left(Y_{0}^{R}(\varrho, t)\right)\right) g^{R}\left(Y_{0}^{R}(\varrho, t)\right)-\left(C^{I}\left(Y_{s}^{I}(\varrho, t)\right)\right. \\
& \left.\left.-C^{I}\left(Y_{0}^{I}(\varrho, t)\right)\right) g^{I}\left(Y_{0}^{I}(\varrho, t)\right)\right) d \varrho+2 \sum_{s=1}^{N} \int_{\Omega}\left(e_{s}^{I}(\varrho, t)\right)^{T}\left(A \triangle e_{s}^{I}(\varrho, t)\right. \\
& -B e_{s}^{I}(\varrho, t)+D^{R}\left(Y_{s}^{R}(\varrho, t)\right) P^{I}\left(e_{s}^{I}(\varrho, t)\right)+D^{I}\left(Y_{s}^{I}(\varrho, t)\right) P^{R}\left(e_{s}^{R}(\varrho, t)\right) \\
& +C^{R}\left(Y_{s}^{R}(\varrho, t)\right) Q^{I}\left(\overline{e_{s}^{I}(\varrho, t)}\right)+x_{s}^{I}(\varrho, t)+C^{I}\left(Y_{s}^{I}(\varrho, t)\right) Q^{R}\left(\overline{e_{s}^{R}(\varrho, t)}\right) \\
& +\hat{h} \sum_{\kappa=1}^{N} \hat{H}_{s \kappa} \hat{M} \triangle e_{\kappa}^{I}(\varrho, t)+\left(D^{R}\left(Y_{s}^{R}(\varrho, t)\right)-D^{R}\left(Y_{0}^{R}(\varrho, t)\right)\right) f^{I}\left(Y_{0}^{I}(\varrho, t)\right) \\
& +\left(D^{I}\left(Y_{s}^{I}(\varrho, t)\right)-D^{I}\left(Y_{0}^{I}(\varrho, t)\right)\right) f^{R}\left(Y_{0}^{R}(\varrho, t)\right)+\left(C^{R}\left(Y_{s}^{R}(\varrho, t)\right)\right. \\
& \left.-C^{R}\left(Y_{0}^{R}(\varrho, t)\right)\right) g^{I}\left(Y_{0}^{I}(\varrho, t)\right)-\Upsilon^{I} e_{s}^{I}(\varrho, t)-\operatorname{sign}\left(e_{s}^{I}(\varrho, t)\right)\left(\bar{D}^{R} \bar{F}^{I}\right. \\
& \left.+\bar{D}^{I} \bar{F}^{R}+\bar{C}^{R} \bar{G}^{I}+\bar{C}^{I} \bar{G}^{R}\right)+\left(C^{I}\left(Y_{s}^{I}(\varrho, t)\right)-C^{I}\left(Y_{0}^{I}(\varrho, t)\right)\right) \\
& \left.\times g^{R}\left(Y_{0}^{R}(\varrho, t)\right)\right) d \varrho+2 \int_{\Omega}\left(e^{R}(\varrho, t)\right)^{T}\left(I_{N} \otimes\left(\zeta^{R} \Gamma\right)\right) e^{R}(\varrho, t) d \varrho \\
& -2 \int_{\Omega}\left(\overline{e^{R}(\varrho, t)}\right)^{T}\left(I_{N} \otimes \zeta^{R}\right) \overline{e^{R}(\varrho, t)} d \varrho+2 \int_{\Omega}\left(e^{I}(\varrho, t)\right)^{T} \\
& \times\left(I_{N} \otimes\left(\zeta^{I} \Gamma\right)\right) e^{I}(\varrho, t) d \varrho-2 \int_{\Omega}\left(\overline{e^{I}(\varrho, t)}\right)^{T}\left(I_{N} \otimes \zeta^{I}\right) \overline{e^{I}(\varrho, t)} d \varrho .
\end{aligned}
$$

On the basis of the boundary condition as well as Green's formula, one has

$$
\begin{aligned}
& 2 \hat{h} \sum_{s=1}^{N} \sum_{k=1}^{N} \int_{\Omega}\left(e_{s}^{R}(\varrho, t)\right)^{T} \hat{H}_{s K} \hat{M} \Delta e_{K}^{R}(\varrho, t) d \varrho \\
= & \hat{h} \int_{\Omega}\left(e^{R}(\varrho, t)\right)^{T}\left(\hat{H} \otimes\left(\hat{M}+\hat{M}^{T}\right)\right) \Delta e^{R}(\varrho, t) d \varrho \\
= & -\hat{h} \sum_{q=1}^{k} \int_{\Omega}\left(\frac{\partial e^{R}(\varrho, t)}{\partial \varrho_{q}}\right)^{T}\left(\hat{H} \otimes\left(\hat{M}+\hat{M}^{T}\right)\right) \frac{\partial e^{R}(\varrho, t)}{\partial \varrho_{q}} d \varrho .
\end{aligned}
$$

Similarly,

$$
2 \hat{h} \sum_{s=1}^{N} \sum_{k=1}^{N} \int_{\Omega}\left(e_{s}^{I}(\varrho, t)\right)^{T} \hat{H}_{s \kappa} \hat{M} \triangle e_{\kappa}^{I}(\varrho, t) d \varrho=-\hat{h} \sum_{q=1}^{k} \int_{\Omega}\left(\frac{\partial e^{I}(\varrho, t)}{\partial \varrho_{q}}\right)^{T}\left(\hat{H} \otimes\left(\hat{M}+\hat{M}^{T}\right)\right) \frac{\partial e^{I}(\varrho, t)}{\partial \varrho_{q}} d \varrho .
$$

Then, we can easily obtain that

$$
\begin{aligned}
& 2 \sum_{s=1}^{N} \int_{\Omega}\left(e_{s}^{R}(\varrho, t)\right)^{T} A \triangle e_{s}^{R}(\varrho, t) d \varrho+2 \hat{h} \sum_{s=1}^{N} \sum_{k=1}^{N} \int_{\Omega}\left(e_{s}^{R}(\varrho, t)\right)^{T} \hat{H}_{s k} \hat{M} \triangle e_{\kappa}^{R}(\varrho, t) d \varrho \\
= & -\sum_{q=1}^{K} \int_{\Omega}\left(\frac{\partial e^{R}(\varrho, t)}{\partial \varrho}\right)^{T}\left(2 I_{N} \otimes A+\hat{h} \hat{H} \otimes\left(\hat{M}+\hat{M}^{T}\right)\right) \frac{\partial e^{R}(\varrho, t)}{\partial \varrho q} d \varrho \\
\leqslant & -\sum_{q=1}^{K} \frac{1}{\xi_{q}^{2}} \int_{\Omega}\left(e^{R}(\varrho, t)\right)^{T}\left(2 I_{N} \otimes A+\hat{h} \hat{H} \otimes\left(\hat{M}+\hat{M}^{T}\right)\right) e^{R}(\varrho, t) d \varrho
\end{aligned}
$$

Similarly,

$$
2 \sum_{s=1}^{N} \int_{\Omega}\left(e_{s}^{I}(\varrho, t)\right)^{T} A \triangle e_{s}^{I}(\varrho, t) d \varrho+2 \hat{h} \sum_{s=1}^{N} \sum_{k=1}^{N} \int_{\Omega}\left(e_{s}^{I}(\varrho, t)\right)^{T} \hat{H}_{s k} \hat{M} \Delta e_{\kappa}^{I}(\varrho, t) d \varrho
$$




$$
\begin{aligned}
& =-\sum_{q=1}^{\kappa} \int_{\Omega}\left(\frac{\partial e^{I}(\varrho, t)}{\partial \varrho_{q}}\right)^{T}\left(2 I_{N} \otimes A+\hat{h} \hat{H} \otimes\left(\hat{M}+\hat{M}^{T}\right)\right) \frac{\partial e^{I}(\varrho, t)}{\partial \varrho_{q}} d \varrho \\
& \leqslant-\sum_{q=1}^{K} \frac{1}{\xi_{q}^{2}} \int_{\Omega}\left(e^{I}(\varrho, t)\right)^{T}\left(2 I_{N} \otimes A+\hat{h} \hat{H} \otimes\left(\hat{M}+\hat{M}^{T}\right)\right) e^{I}(\varrho, t) d \varrho .
\end{aligned}
$$

From (12)-(19), (22)-(29), (46) and (47), we have

$$
\begin{aligned}
\dot{V}(t) \leqslant & \int_{\Omega}\left(e^{R}(\varrho, t)\right)^{T}\left(I_{N} \otimes\left(-\sum_{q=1}^{K} \frac{2 A}{\xi_{q}^{2}}-2 B+\tilde{D}^{R}+2 L^{R}+\tilde{D}^{I}+\tilde{C}^{R}+\tilde{C}^{I}-\Upsilon^{R}+2 \zeta^{R} \Gamma\right)-\sum_{q=1}^{\kappa} \frac{\hat{h}}{\xi_{q}^{2}} \hat{H} \otimes\left(\hat{M}+\hat{M}^{T}\right)\right) e^{R}(\varrho, t) d \varrho \\
& \left.+\int_{\Omega}\left(e^{I}(\varrho, t)\right)^{T}\left(I_{N} \otimes\left(-\sum_{q=1}^{\kappa} \frac{2 A}{\xi_{q}^{2}}-2 B+2 L^{I}+\tilde{D}^{R}+\tilde{D}^{I}+\tilde{C}^{I}+\tilde{C}^{R}-\Upsilon^{I}+2 \zeta^{I} \Gamma\right)\right)-\sum_{q=1}^{\kappa} \frac{\hat{h}}{\xi_{q}^{2}} \hat{H} \otimes\left(\hat{M}+\hat{M}^{T}\right)\right) e^{I}(\varrho, t) d \varrho \\
& +2 \int_{\Omega}\left(e^{R}(\varrho, t)\right)^{T} x^{R}(\varrho, t) d \varrho+2 \int_{\Omega}\left(e^{I}(\varrho, t)\right)^{T} x^{I}(\varrho, t) d \varrho .
\end{aligned}
$$

Furthermore,

$$
\begin{aligned}
& \dot{V}(t)-\int_{\Omega}\left[\left(z^{R}(\varrho, t)\right)^{T} x^{R}(\varrho, t)+\left(z^{I}(\varrho, t)\right)^{T} x^{I}(\varrho, t)\right] d \varrho-\rho \int_{\Omega}\left[\left(x^{R}(\varrho, t)\right)^{T} x^{R}(\varrho, t)+\left(x^{I}(\varrho, t)\right)^{T} x^{I}(\varrho, t)\right] d \varrho \\
\leqslant & \int_{\Omega}\left(e^{R}(\varrho, t)\right)^{T}\left(I_{N} \otimes\left(-\sum_{q=1}^{K} \frac{2 A}{\xi_{q}^{2}}-2 B+\tilde{D}^{R}+2 L^{R}+\tilde{D}^{I}+\tilde{C}^{R}+\tilde{C}^{I}-\Upsilon^{R}+2 \zeta^{R} \Gamma\right)-\sum_{q=1}^{\kappa} \frac{\hat{h}}{\xi_{q}^{2}} \hat{H} \otimes(\hat{M}\right. \\
& \left.\left.+\hat{M}^{T}\right)\right) e^{R}(\varrho, t) d \varrho+\int_{\Omega}\left(e^{I}(\varrho, t)\right)^{T}\left(I _ { N } \otimes \left(-\sum_{q=1}^{\kappa} \frac{2 A}{\xi_{q}^{2}}-2 B+2 L^{I}+\tilde{D}^{R}+\tilde{D}^{I}+\tilde{C}^{I}+\tilde{C}^{R}-\Upsilon^{I}\right.\right. \\
& \left.\left.+2 \zeta^{I} \Gamma\right)-\sum_{q=1}^{\kappa} \frac{\hat{h}}{\xi_{q}^{2}} \hat{H} \otimes\left(\hat{M}+\hat{M}^{T}\right)\right) e^{I}(\varrho, t) d \varrho+\int_{\Omega}\left(x^{R}(\varrho, t)\right)^{T}\left(I_{N} \otimes\left(-\frac{1}{2}\left(\hat{K}_{2}^{T}+\hat{K}_{2}\right)-\rho I_{n}\right)\right) x^{R}(\varrho, t) d \varrho \\
& +\int_{\Omega}\left(x^{I}(\varrho, t)\right)^{T}\left(I_{N} \otimes\left(-\frac{1}{2}\left(\hat{K}_{2}^{T}+\hat{K}_{2}\right)-\rho I_{n}\right)\right) x^{I}(\varrho, t) d \varrho+2 \int_{\Omega}\left(e^{R}(\varrho, t)\right)^{T}\left(I _ { N } \otimes \left(I_{n}\right.\right. \\
& \left.\left.-\frac{1}{2} \hat{K}_{1}^{T}\right)\right) x^{R}(\varrho, t) d \varrho+2 \int_{\Omega}\left(e^{I}(\varrho, t)\right)^{T}\left(I_{N} \otimes\left(I_{n}-\frac{1}{2} \hat{K}_{1}^{T}\right)\right) x^{I}(\varrho, t) d \varrho \\
= & \int_{\Omega}\left(\varphi^{R}(\varrho, t)\right)^{T}\left(\begin{array}{cc}
\hat{\Psi}_{1}^{R} & \hat{\Xi}^{R} \\
\left(\hat{\Xi}^{R}\right)^{T} & \hat{\Psi}_{2}^{R}
\end{array}\right) \varphi^{R}(\varrho, t) d \varrho+\int_{\Omega}\left(\varphi^{I}(\varrho, t)\right)^{T}\left(\begin{array}{l}
\hat{\Psi}_{1}^{I} \\
\left(\hat{\Xi}^{I}\right)^{T}
\end{array} \hat{\Psi}_{2}^{I}\right) \varphi^{I}(\varrho, t) d \varrho,
\end{aligned}
$$

where $\varphi^{R}(\varrho, t)=\left(\left(e^{R}(\varrho, t)\right)^{T},\left(x^{R}(\varrho, t)\right)^{T}\right)^{T}$ and $\varphi^{I}(\varrho, t)=\left(\left(e^{I}(\varrho, t)\right)^{T},\left(x^{I}(\varrho, t)\right)^{T}\right)^{T}$. From (43) and (44), we can easily obtain

$$
\dot{V}(t) \leqslant \int_{\Omega}\left[\left(z^{R}(\varrho, t)\right)^{T} x^{R}(\varrho, t)+\left(z^{I}(\varrho, t)\right)^{T} x^{I}(\varrho, t)\right] d \varrho+\rho \int_{\Omega}\left[\left(x^{R}(\varrho, t)\right)^{T} x^{R}(\varrho, t)+\left(x^{I}(\varrho, t)\right)^{T} x^{I}(\varrho, t)\right] d \varrho .
$$

By integrating (48) about $t$ over the time period from $t_{1}$ to $t_{2}$, one has

$$
\begin{aligned}
V\left(t_{2}\right)-V\left(t_{1}\right) \leqslant & \int_{t_{1}}^{t_{2}} \int_{\Omega}\left[\left(z^{R}(\varrho, t)\right)^{T} x^{R}(\varrho, t)+\left(z^{I}(\varrho, t)\right)^{T} x^{I}(\varrho, t)\right] d \varrho d t \\
& +\rho \int_{t_{1}}^{t_{2}} \int_{\Omega}\left[\left(x^{R}(\varrho, t)\right)^{T} x^{R}(\varrho, t)+\left(x^{I}(\varrho, t)\right)^{T} x^{I}(\varrho, t)\right] d \varrho d t, t_{2} \geqslant t_{1} .
\end{aligned}
$$

Namely,

$$
\int_{t_{1}}^{t_{2}} \int_{\Omega}\left[\left(z^{R}(\varrho, t)\right)^{T} x^{R}(\varrho, t)+\left(z^{I}(\varrho, t)\right)^{T} x^{I}(\varrho, t)\right] d \varrho d t \geqslant V\left(t_{2}\right)-V\left(t_{1}\right)-\rho \int_{t_{1}}^{t_{2}} \int_{\Omega}\left[\left(x^{R}(\varrho, t)\right)^{T} x^{R}(\varrho, t)+\left(x^{I}(\varrho, t)\right)^{T} x^{I}(\varrho, t)\right] d \varrho d t
$$
for any $t_{2}, t_{1} \in \mathbb{R}^{+}$and $t_{2} \geqslant t_{1}$.

According to Definition 3.1, we can obtain that the network (42) is passive under the controller (7). 


\subsection{Synchronization control}

Theorem 4.2. The network (39) is said to be synchronized under the controller (7) if

$$
2 I_{N} \otimes A+\hat{h} \hat{H} \otimes\left(\hat{M}+\hat{M}^{T}\right) \geqslant 0, \quad \hat{\Psi}_{1}^{R}<0 \text { and } \hat{\Psi}_{1}^{I}<0,
$$

where $\hat{\Psi}_{1}^{R}=I_{N} \otimes\left(-\sum_{q=1}^{K} \frac{2 A}{\xi_{q}^{2}}-2 B+\tilde{D}^{R}+2 L^{R}+\tilde{D}^{I}+\tilde{C}^{R}+\tilde{C}^{I}-\Upsilon^{R}+2 \zeta^{R} \Gamma\right)-\sum_{q=1}^{\kappa} \frac{\hat{h}}{\xi_{q}^{2}} \hat{H} \otimes\left(\hat{M}+\hat{M}^{T}\right), \hat{\Psi}_{1}^{I}=$ $I_{N} \otimes\left(-\sum_{q=1}^{\kappa} \frac{2 A}{\xi_{q}^{2}}-2 B+2 L^{I}+\tilde{D}^{R}+\tilde{D}^{I}+\tilde{C}^{I}+\tilde{C}^{R}-\Upsilon^{I}+2 \zeta^{I} \Gamma\right)-\sum_{q=1}^{\kappa} \frac{\hat{h}}{\xi_{q}^{2}} \hat{H} \otimes\left(\hat{M}+\hat{M}^{T}\right)$.

Proof. Define the same Lyapunov functional for network (42) as in Theorem 3.1. Then, one has

$$
\begin{aligned}
\dot{V}(t) \leqslant & \int_{\Omega}\left(e^{R}(\varrho, t)\right)^{T}\left(I _ { N } \otimes \left(-\sum_{q=1}^{\kappa} \frac{2 A}{\xi_{q}^{2}}-2 B+\tilde{D}^{R}+2 L^{R}+\tilde{D}^{I}+\tilde{C}^{R}+\tilde{C}^{I}-\Upsilon^{R}\right.\right. \\
& \left.\left.+2 \zeta^{R} \Gamma\right)-\sum_{q=1}^{\kappa} \frac{\hat{h}}{\xi_{q}^{2}} \hat{H} \otimes\left(\hat{M}+\hat{M}^{T}\right)\right) e^{R}(\varrho, t) d \varrho+\int_{\Omega}\left(e^{I}(\varrho, t)\right)^{T}\left(I _ { N } \otimes \left(-\sum_{q=1}^{\kappa} \frac{2 A}{\xi_{q}^{2}}\right.\right. \\
& \left.-2 B+2 L^{I}+\tilde{D}^{R}+\tilde{D}^{I}+\tilde{C}^{I}+\tilde{C}^{R}-\Upsilon^{I}+2 \zeta^{I} \Gamma\right)-\sum_{q=1}^{\kappa} \frac{\hat{h}}{\xi_{q}^{2}} \hat{H} \otimes(\hat{M} \\
& \left.\left.+\hat{M}^{T}\right)\right) e^{I}(\varrho, t) d \varrho \\
\leqslant & \hat{\alpha}\|e(\cdot, t)\|^{2},
\end{aligned}
$$

where $\hat{\alpha}=\max \left\{\lambda_{M}\left(\hat{\Psi}_{1}^{R}\right), \lambda_{M}\left(\hat{\Psi}_{1}^{I}\right)\right\}$.

Similar to the proof of Theorem 3.2, we can easily obtain $\lim _{t \rightarrow+\infty}\|e(\cdot, t)\|=0$. Therefore, the network (39) is synchronized.

\section{Numerical Examples}

Example 5.1. Consider the following CRDCVMNN:

$$
\begin{aligned}
\frac{\partial Y_{s}(\varrho, t)}{\partial t}= & A \triangle Y_{s}(\varrho, t)-B Y_{s}(\varrho, t)+C\left(Y_{s}(\varrho, t)\right) g\left(\overline{Y_{s}(\varrho, t)}\right)+u_{s}(\varrho, t)+D\left(Y_{s}(\varrho, t)\right) f\left(Y_{s}(\varrho, t)\right) \\
& +h \sum_{\kappa=1}^{N} H_{s K} M Y_{\kappa}(\varrho, t)+x_{s}(\varrho, t),
\end{aligned}
$$

where $s=1,2, \cdots, 6, f_{i}^{R}(\omega)=f_{i}^{I}(\omega)=g_{i}^{R}(\omega)=g_{i}^{I}(\omega)=\frac{|\omega+1|-|\omega-1|}{4}(i=1,2,3), \Omega=\{\varrho \mid-1<\varrho<1\}, A=$ $\operatorname{diag}(0.6,0.8,0.7), B=\operatorname{diag}(0.8,0.9,0.8), M=\operatorname{diag}(0.6,0.5,0.3), h=0.1, \tau_{j}(t)=1-\frac{1}{2+j} e^{-t}, \tau=1, \gamma_{j}=\frac{1}{2+j}$, $j=1,2,3$, and the matrices $C\left(Y_{s}(\varrho, t)\right), D\left(Y_{s}(\varrho, t)\right), H=\left(H_{s K}\right)_{6 \times 6}$ are selected as follows:

$$
\begin{gathered}
c_{11}^{R}\left(y_{s 1}^{R}(\varrho, t)\right)=\left\{\begin{array}{ll}
-0.35, & \left|y_{s 1}^{R}(\varrho, t)\right| \leqslant 1.1, \\
-0.25, & \left|y_{s 1}^{R}(\varrho, t)\right|>1.1,
\end{array} \quad c_{12}^{R}\left(y_{s 1}^{R}(\varrho, t)\right)= \begin{cases}0.35, & \left|y_{s 1}^{R}(\varrho, t)\right| \leqslant 1.1, \\
-0.45, & \left|y_{s 1}^{R}(\varrho, t)\right|>1.1,\end{cases} \right. \\
c_{13}^{R}\left(y_{s 1}^{R}(\varrho, t)\right)=\left\{\begin{array}{ll}
0.32, & \left|y_{s 1}^{R}(\varrho, t)\right| \leqslant 1.1, \\
0.33, & \left|y_{s 1}^{R}(\varrho, t)\right|>1.1,
\end{array} \quad c_{21}^{R}\left(y_{s 2}^{R}(\varrho, t)\right)= \begin{cases}0.43, & \left|y_{s 2}^{R}(\varrho, t)\right| \leqslant 1.1, \\
0.25, & \left|y_{s 2}^{R}(\varrho, t)\right|>1.1,\end{cases} \right. \\
c_{22}^{R}\left(y_{s 2}^{R}(\varrho, t)\right)=\left\{\begin{array}{ll}
-0.24, & \left|y_{s 2}^{R}(\varrho, t)\right| \leqslant 1.1, \\
0.15, & \left|y_{s 2}^{R}(\varrho, t)\right|>1.1,
\end{array} \quad c_{23}^{R}\left(y_{s 2}^{R}(\varrho, t)\right)= \begin{cases}-0.12, & \left|y_{s 2}^{R}(\varrho, t)\right| \leqslant 1.1, \\
0.11, & \left|y_{s 2}^{R}(\varrho, t)\right|>1.1,\end{cases} \right. \\
c_{31}^{R}\left(y_{s 3}^{R}(\varrho, t)\right)=\left\{\begin{array}{ll}
-0.15, & \left|y_{s 3}^{R}(\varrho, t)\right| \leqslant 1.1, \\
-0.25, & \left|y_{s 3}^{R}(\varrho, t)\right|>1.1,
\end{array} \quad c_{32}^{R}\left(y_{s 3}^{R}(\varrho, t)\right)= \begin{cases}0.33, & \left|y_{s 3}^{R}(\varrho, t)\right| \leqslant 1.1, \\
0.25, & \left|y_{s 3}^{R}(\varrho, t)\right|>1.1,\end{cases} \right. \\
c_{33}^{R}\left(y_{s 3}^{R}(\varrho, t)\right)=\left\{\begin{array}{ll}
0.15, & \left|y_{s 3}^{R}(\varrho, t)\right| \leqslant 1.1, \\
0.21, & \left|y_{s 3}^{R}(\varrho, t)\right|>1.1,
\end{array} \quad c_{11}^{I}\left(y_{s 1}^{I}(\varrho, t)\right)= \begin{cases}-0.15, & \left|y_{s 1}^{I}(\varrho, t)\right| \leqslant 1.1, \\
-0.27, & \left|y_{s 1}^{I}(\varrho, t)\right|>1.1,\end{cases} \right.
\end{gathered}
$$




$$
\begin{aligned}
& c_{12}^{I}\left(y_{s 1}^{I}(\varrho, t)\right)=\left\{\begin{array}{ll}
0.25, & \left|y_{s 1}^{I}(\varrho, t)\right| \leqslant 1.1, \\
0.15, & \left|y_{s 1}^{I}(\varrho, t)\right|>1.1,
\end{array} \quad c_{13}^{I}\left(y_{s 1}^{I}(\varrho, t)\right)= \begin{cases}0.32, & \left|y_{s 1}^{I}(\varrho, t)\right| \leqslant 1.1, \\
0.13, & \left|y_{s 1}^{I}(\varrho, t)\right|>1.1,\end{cases} \right. \\
& c_{21}^{I}\left(y_{s 2}^{I}(\varrho, t)\right)=\left\{\begin{array}{ll}
0.42, & \left|y_{s 2}^{I}(\varrho, t)\right| \leqslant 1.1, \\
0.25, & \left|y_{s 2}^{I}(\varrho, t)\right|>1.1,
\end{array} \quad c_{22}^{I}\left(y_{s 2}^{I}(\varrho, t)\right)=\left\{\begin{array}{l}
-0.14, \quad\left|y_{s 2}^{I}(\varrho, t)\right| \leqslant 1.1, \\
0.12, \quad\left|y_{s 2}^{I}(\varrho, t)\right|>1.1,
\end{array}\right.\right. \\
& c_{23}^{I}\left(y_{s 2}^{I}(\varrho, t)\right)=\left\{\begin{array}{l}
0.22, \quad\left|y_{s 2}^{I}(\varrho, t)\right| \leqslant 1.1, \\
-0.11, \quad\left|y_{s 2}^{I}(\varrho, t)\right|>1.1,
\end{array} \quad c_{31}^{I}\left(y_{s 3}^{I}(\varrho, t)\right)=\left\{\begin{array}{l}
0.15, \quad\left|y_{s 3}^{I}(\varrho, t)\right| \leqslant 1.1, \\
-0.23, \quad\left|y_{s 3}^{I}(\varrho, t)\right|>1.1,
\end{array}\right.\right. \\
& c_{32}^{I}\left(y_{s 3}^{I}(\varrho, t)\right)=\left\{\begin{array}{ll}
0.34, & \left|y_{s 3}^{I}(\varrho, t)\right| \leqslant 1.1, \\
0.25, & \left|y_{s 3}^{I}(\varrho, t)\right|>1.1,
\end{array} \quad c_{33}^{I}\left(y_{s 3}^{I}(\varrho, t)\right)= \begin{cases}0.31, & \left|y_{s 3}^{I}(\varrho, t)\right| \leqslant 1.1, \\
0.25, & \left|y_{s 3}^{I}(\varrho, t)\right|>1.1,\end{cases} \right. \\
& d_{11}^{R}\left(y_{s 1}^{R}(\varrho, t)\right)=\left\{\begin{array}{ll}
-0.34, & \left|y_{s 1}^{R}(\varrho, t)\right| \leqslant 1.1, \\
-0.25, & \left|y_{s 1}^{R}(\varrho, t)\right|>1.1,
\end{array} \quad d_{12}^{R}\left(y_{s 1}^{R}(\varrho, t)\right)= \begin{cases}0.25, & \left|y_{s 1}^{R}(\varrho, t)\right| \leqslant 1.1, \\
-0.15, & \left|y_{s 1}^{R}(\varrho, t)\right|>1.1,\end{cases} \right. \\
& d_{13}^{R}\left(y_{s 1}^{R}(\varrho, t)\right)=\left\{\begin{array}{ll}
0.14, & \left|y_{s 1}^{R}(\varrho, t)\right| \leqslant 1.1, \\
0.13, & \left|y_{s 1}^{R}(\varrho, t)\right|>1.1,
\end{array} \quad d_{21}^{R}\left(y_{s 2}^{R}(\varrho, t)\right)= \begin{cases}0.43, & \left|y_{s 2}^{R}(\varrho, t)\right| \leqslant 1.1, \\
0.45, & \left|y_{s 2}^{R}(\varrho, t)\right|>1.1,\end{cases} \right. \\
& d_{22}^{R}\left(y_{s 2}^{R}(\varrho, t)\right)=\left\{\begin{array}{l}
-0.25, \quad\left|y_{s 2}^{R}(\varrho, t)\right| \leqslant 1.1, \\
0.15, \quad\left|y_{s 2}^{R}(\varrho, t)\right|>1.1,
\end{array} \quad d_{23}^{R}\left(y_{s 2}^{R}(\varrho, t)\right)=\left\{\begin{array}{l}
-0.12, \quad\left|y_{s 2}^{R}(\varrho, t)\right| \leqslant 1.1, \\
0.13, \quad\left|y_{s 2}^{R}(\varrho, t)\right|>1.1,
\end{array}\right.\right. \\
& d_{31}^{R}\left(y_{s 3}^{R}(\varrho, t)\right)=\left\{\begin{array}{ll}
-0.15, & \left|y_{s 3}^{R}(\varrho, t)\right| \leqslant 1.1, \\
-0.26, & \left|y_{s 3}^{R}(\varrho, t)\right|>1.1,
\end{array} \quad d_{32}^{R}\left(y_{s 3}^{R}(\varrho, t)\right)= \begin{cases}0.13, & \left|y_{s 3}^{R}(\varrho, t)\right| \leqslant 1.1, \\
0.11, & \left|y_{s 3}^{R}(\varrho, t)\right|>1.1,\end{cases} \right. \\
& d_{33}^{R}\left(y_{s 3}^{R}(\varrho, t)\right)=\left\{\begin{array}{ll}
0.15, \quad\left|y_{s 3}^{R}(\varrho, t)\right| \leqslant 1.1, \\
-0.24, \quad\left|y_{s 3}^{R}(\varrho, t)\right|>1.1,
\end{array} \quad d_{11}^{I}\left(y_{s 1}^{I}(\varrho, t)\right)= \begin{cases}-0.15, & \left|y_{s 1}^{I}(\varrho, t)\right| \leqslant 1.1, \\
-0.25, & \left|y_{s 1}^{I}(\varrho, t)\right|>1.1,\end{cases} \right. \\
& d_{12}^{I}\left(y_{s 1}^{I}(\varrho, t)\right)=\left\{\begin{array}{ll}
0.24, & \left|y_{s 1}^{I}(\varrho, t)\right| \leqslant 1.1, \\
0.15, & \left|y_{s 1}^{I}(\varrho, t)\right|>1.1,
\end{array} \quad d_{13}^{I}\left(y_{s 1}^{I}(\varrho, t)\right)= \begin{cases}0.31, & \left|y_{s 1}^{I}(\varrho, t)\right| \leqslant 1.1, \\
0.13, & \left|y_{s 1}^{I}(\varrho, t)\right|>1.1,\end{cases} \right. \\
& d_{21}^{I}\left(y_{s 2}^{I}(\varrho, t)\right)=\left\{\begin{array}{ll}
0.32, & \left|y_{s 2}^{I}(\varrho, t)\right| \leqslant 1.1, \\
0.25, & \left|y_{s 2}^{I}(\varrho, t)\right|>1.1,
\end{array} \quad d_{22}^{I}\left(y_{s 2}^{I}(\varrho, t)\right)=\left\{\begin{array}{l}
-0.34, \quad\left|y_{s 2}^{I}(\varrho, t)\right| \leqslant 1.1, \\
0.16, \quad\left|y_{s 2}^{I}(\varrho, t)\right|>1.1,
\end{array}\right.\right. \\
& d_{23}^{I}\left(y_{s 2}^{I}(\varrho, t)\right)=\left\{\begin{array}{l}
0.12, \quad\left|y_{s 2}^{I}(\varrho, t)\right| \leqslant 1.1, \\
-0.11, \quad\left|y_{s 2}^{I}(\varrho, t)\right|>1.1,
\end{array} \quad d_{31}^{I}\left(y_{s 3}^{I}(\varrho, t)\right)=\left\{\begin{array}{l}
0.21, \quad\left|y_{s 3}^{I}(\varrho, t)\right| \leqslant 1.1, \\
-0.16, \quad\left|y_{s 3}^{I}(\varrho, t)\right|>1.1,
\end{array}\right.\right. \\
& d_{32}^{I}\left(y_{s 3}^{I}(\varrho, t)\right)=\left\{\begin{array}{ll}
0.24, & \left|y_{s 3}^{I}(\varrho, t)\right| \leqslant 1.1, \\
0.12, & \left|y_{s 3}^{I}(\varrho, t)\right|>1.1,
\end{array} \quad d_{33}^{I}\left(y_{s 3}^{I}(\varrho, t)\right)=\left\{\begin{array}{l}
-0.35, \quad\left|y_{s 3}^{I}(\varrho, t)\right| \leqslant 1.1, \\
0.25, \quad\left|y_{s 3}^{I}(\varrho, t)\right|>1.1,
\end{array}\right.\right.
\end{aligned}
$$

$$
H=\left(\begin{array}{cccccc}
-0.6 & 0.3 & 0.1 & 0 & 0.1 & 0.1 \\
0.3 & -0.7 & 0.2 & 0 & 0.1 & 0.1 \\
0.1 & 0.2 & -1.0 & 0.1 & 0.5 & 0.1 \\
0 & 0 & 0.1 & -0.4 & 0.2 & 0.1 \\
0.1 & 0.1 & 0.5 & 0.2 & -1.0 & 0.1 \\
0.1 & 0.1 & 0.1 & 0.1 & 0.1 & -0.5
\end{array}\right) .
$$

Apparently, $f_{i}^{R}(\cdot), f_{i}^{I}(\cdot), g_{i}^{R}(\cdot)$ and $g_{i}^{I}(\cdot)(i=1,2,3)$ satisfy Assumption 1 with $F_{i}^{R}=F_{i}^{I}=G_{s}^{R}=G_{s}^{I}=0.5$ and $l_{s}^{R}=l_{s}^{I}=\eta_{s}^{R}=\eta_{s}^{I}=0.5$. The input $x_{s 1}(\varrho, t)=0.5 s \sqrt{t} \cos (\pi \varrho)+i 0.4 s \sqrt{t} \cos (\pi \varrho), x_{s 2}(\varrho, t)=0.1 s \sqrt{t} \cos (\pi \varrho)+$ $i 0.2 s \sqrt{t} \cos (\pi \varrho), x_{s 3}(\varrho, t)=0.3 s \sqrt{t} \cos (\pi \varrho)+i 0.2 s \sqrt{t} \cos (\pi \varrho)$. The parameters in the controller (7) are chosen as follows: $\Upsilon^{R}=\operatorname{diag}(0.8,0.9,0.7), \Upsilon^{I}=\operatorname{diag}(0.7,1.1,0.9)$. Take $K_{1}$ and $K_{2}$ as follows:

$$
K_{1}=\left(\begin{array}{ccc}
0 & 0.3 & 0 \\
0.2 & -0.5 & 0.1 \\
0.4 & 0 & -0.3
\end{array}\right), K_{2}=\left(\begin{array}{ccc}
-0.1 & 0.3 & 0.2 \\
0 & -0.4 & 0.1 \\
0.2 & 0.3 & -0.3
\end{array}\right)
$$




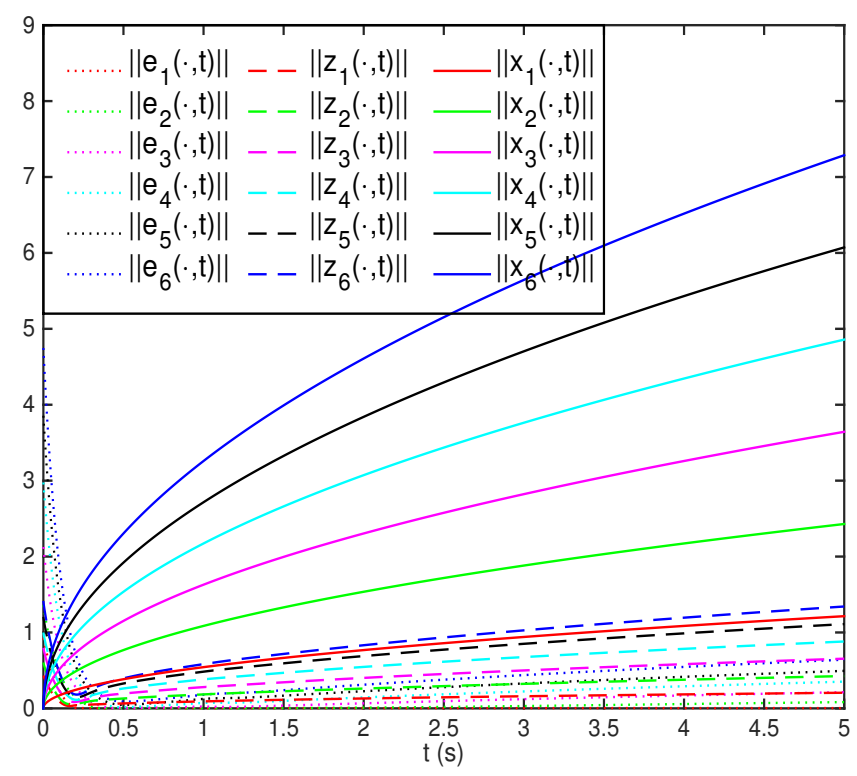

Figure 1: The norms of $e_{s}(\cdot, t), z_{s}(\cdot, t), x_{s}(\cdot, t), s=1,2, \cdots, 6$.

By exploiting the MATLAB, we can easily obtain that $\rho=4.9466$ which satisfies the condition (8). According to Theorem 3.1, the system (50) is passive under controller (7). Figure 1 respectively shows the evolutions of error, output and input of six nodes when the system (50) is passive. Similarly, through a simple operation based on the above parameters by using MATLAB, we have

$$
\begin{aligned}
\lambda\left(\Psi_{1}^{R}\right)= & \{-2.2689,-2.2144,-2.1928,-2.1777,-2.1549,-2.1177,-1.8193,-1.7866,-1.7736,-1.7646,-1.7509, \\
& -1.7286,-1.4458,-1.3804,-1.3545,-1.3364,-1.3090,-1.2644\}, \\
\lambda\left(\Psi_{1}^{I}\right)= & \{-2.4689,-2.4144,-2.3928,-2.3777,-2.3549,-2.3177,-2.0193,-1.9866,-1.9736,-1.9646,-1.9509, \\
& -1.9286,-1.3458,-1.2804,-1.2545,-1.2364,-1.2090,-1.1644\},
\end{aligned}
$$

which satisfy the condition (31). On the basis of Theorem 3.2, the system (50) realizes synchronization. Figure 2 depicts the simulation result of synchronization.

Example 5.2. Consider the following CRDCVMNN with spatial diffusion coupling:

$$
\begin{aligned}
\frac{\partial Y_{s}(\varrho, t)}{\partial t}= & A \Delta Y_{s}(\varrho, t)-B Y_{s}(\varrho, t)+C\left(Y_{s}(\varrho, t)\right) g\left(\overline{Y_{s}(\varrho, t)}\right)+u_{s}(\varrho, t)+D\left(Y_{s}(\varrho, t)\right) f\left(Y_{s}(\varrho, t)\right) \\
& +\hat{h} \sum_{k=1}^{N} \hat{H}_{s k} \hat{M} \triangle Y_{K}(\varrho, t)+x_{s}(\varrho, t),
\end{aligned}
$$

where $s=1,2, \cdots, 6, f_{i}^{R}(\omega)=f_{i}^{I}(\omega)=g_{i}^{R}(\omega)=g_{i}^{I}(\omega)=\frac{|\omega+1|-|\omega-1|}{8}(i=1,2,3), \Omega=\{\varrho \mid-1<\varrho<1\}, A=$ $\operatorname{diag}(0.7,0.6,0.7), B=\operatorname{diag}(0.8,0.9,0.8), \hat{M}=\operatorname{diag}(0.7,0.6,0.4), \hat{h}=0.2, \tau_{j}(t)=1-\frac{1}{3 j} e^{-t}, \tau=1, \gamma_{j}=\frac{1}{3 j}$, $j=1,2,3$, and the matrices $C\left(Y_{s}(\varrho, t)\right), D\left(Y_{s}(\varrho, t)\right), \hat{H}=\left(\hat{H}_{s K}\right)_{6 \times 6}$ are selected as follows:

$$
\begin{aligned}
& c_{11}^{R}\left(y_{s 1}^{R}(\varrho, t)\right)=\left\{\begin{array}{ll}
0.45, & \left|y_{s 1}^{R}(\varrho, t)\right| \leqslant 1.3, \\
-0.35, & \left|y_{s 1}^{R}(\varrho, t)\right|>1.3,
\end{array} \quad c_{12}^{R}\left(y_{s 1}^{R}(\varrho, t)\right)= \begin{cases}-0.35, & \left|y_{s 1}^{R}(\varrho, t)\right| \leqslant 1.3, \\
-0.25, & \left|y_{s 1}^{R}(\varrho, t)\right|>1.3,\end{cases} \right. \\
& c_{13}^{R}\left(y_{s 1}^{R}(\varrho, t)\right)=\left\{\begin{array}{ll}
0.12, & \left|y_{s 1}^{R}(\varrho, t)\right| \leqslant 1.3, \\
0.35, & \left|y_{s 1}^{R}(\varrho, t)\right|>1.3,
\end{array} \quad c_{21}^{R}\left(y_{s 2}^{R}(\varrho, t)\right)= \begin{cases}-0.23, & \left|y_{s 2}^{R}(\varrho, t)\right| \leqslant 1.3, \\
0.25, & \left|y_{s 2}^{R}(\varrho, t)\right|>1.3,\end{cases} \right.
\end{aligned}
$$




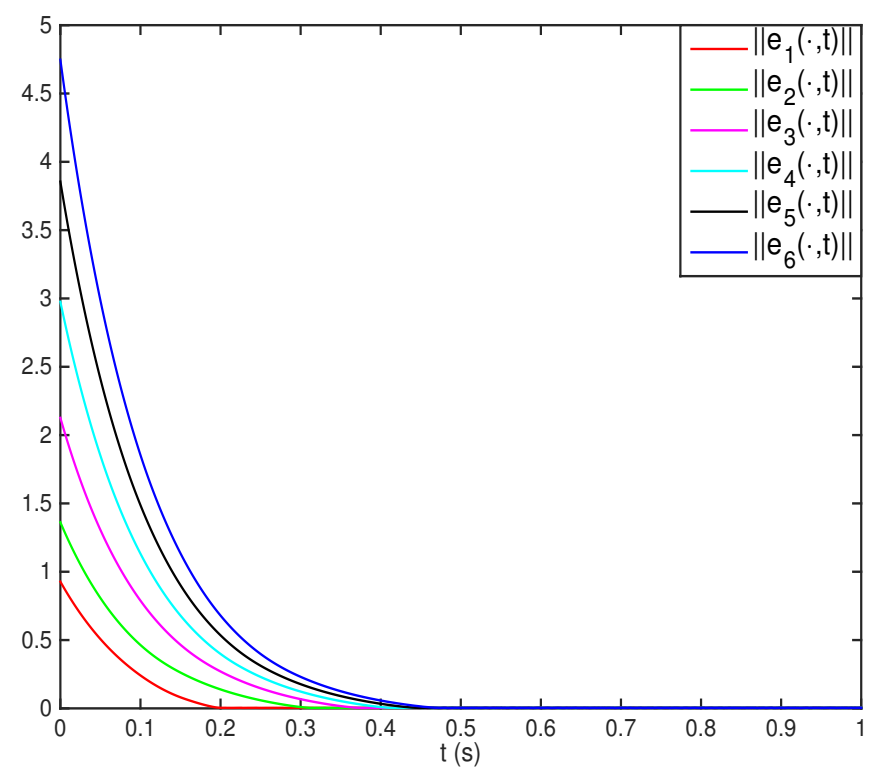

Figure 2: Time evolutions of $e_{s}(\cdot, t), s=1,2, \cdots, 6$.

$$
\begin{gathered}
c_{22}^{R}\left(y_{s 2}^{R}(\varrho, t)\right)=\left\{\begin{array}{ll}
0.14, & \left|y_{s 2}^{R}(\varrho, t)\right| \leqslant 1.3, \\
0.26, & \left|y_{s 2}^{R}(\varrho, t)\right|>1.3,
\end{array} \quad c_{23}^{R}\left(y_{s 2}^{R}(\varrho, t)\right)= \begin{cases}-0.22, & \left|y_{s 2}^{R}(\varrho, t)\right| \leqslant 1.3, \\
0.11, & \left|y_{s 2}^{R}(\varrho, t)\right|>1.3,\end{cases} \right. \\
c_{31}^{R}\left(y_{s 3}^{R}(\varrho, t)\right)=\left\{\begin{array}{ll}
0.13, & \left|y_{s 3}^{R}(\varrho, t)\right| \leqslant 1.3, \\
0.25, & \left|y_{s 3}^{R}(\varrho, t)\right|>1.3,
\end{array} \quad c_{32}^{R}\left(y_{s 3}^{R}(\varrho, t)\right)= \begin{cases}0.23, & \left|y_{s 3}^{R}(\varrho, t)\right| \leqslant 1.3, \\
0.25, & \left|y_{s 3}^{R}(\varrho, t)\right|>1.3,\end{cases} \right. \\
c_{33}^{R}\left(y_{s 3}^{R}(\varrho, t)\right)=\left\{\begin{array}{ll}
0.16, & \left|y_{s 3}^{R}(\varrho, t)\right| \leqslant 1.3, \\
-0.11, & \left|y_{s 3}^{R}(\varrho, t)\right|>1.3,
\end{array} \quad c_{11}^{I}\left(y_{s 1}^{I}(\varrho, t)\right)= \begin{cases}-0.15, & \left|y_{s 1}^{I}(\varrho, t)\right| \leqslant 1.3, \\
-0.26, & \left|y_{s 1}^{I}(\varrho, t)\right|>1.3,\end{cases} \right. \\
c_{12}^{I}\left(y_{s 1}^{I}(\varrho, t)\right)=\left\{\begin{array}{ll}
0.24, & \left|y_{s 1}^{I}(\varrho, t)\right| \leqslant 1.3, \\
0.31, & \left|y_{s 1}^{I}(\varrho, t)\right|>1.3,
\end{array} \quad c_{13}^{I}\left(y_{s 1}^{I}(\varrho, t)\right)= \begin{cases}-0.12, & \left|y_{s 1}^{I}(\varrho, t)\right| \leqslant 1.3, \\
0.13, & \left|y_{s 1}^{I}(\varrho, t)\right|>1.3,\end{cases} \right. \\
c_{21}^{I}\left(y_{s 2}^{I}(\varrho, t)\right)=\left\{\begin{array}{ll}
0.32, & \left|y_{s 2}^{I}(\varrho, t)\right| \leqslant 1.3, \\
-0.25, & \left|y_{s 2}^{I}(\varrho, t)\right|>1.3,
\end{array} \quad c_{22}^{I}\left(y_{s 2}^{I}(\varrho, t)\right)= \begin{cases}0.15, & \left|y_{s 2}^{I}(\varrho, t)\right| \leqslant 1.3, \\
0.12, & \left|y_{s 2}^{I}(\varrho, t)\right|>1.3,\end{cases} \right. \\
c_{32}^{I}\left(y_{s 3}^{I}(\varrho, t)\right)=\left\{\begin{array}{ll}
0.34, & \left|y_{s 3}^{I}(\varrho, t)\right| \leqslant 1.3, \\
0.26, & \left|y_{s 3}^{I}(\varrho, t)\right|>1.3, \\
-0.11, & \left|y_{s 2}^{I}(\varrho, t)\right|>1.3,
\end{array} \quad c_{33}^{I}\left(y_{s 3}^{I}(\varrho, t)\right)= \begin{cases}-0.22, & \left|y_{s 3}^{I}(\varrho, t)\right| \leqslant 1.3, \\
-0.25, & \left|y_{s 3}^{I}(\varrho, t)\right|>1.3, \\
0.13, & \left|y_{s 3}^{I}(\varrho, t)\right|>1.3,\end{cases} \right. \\
d_{11}^{R}\left(y_{s 1}^{R}(\varrho, t)\right)=\left\{\begin{array}{ll}
-0.36, & \left|y_{s 1}^{R}(\varrho, t)\right| \leqslant 1.3, \\
-0.25, & \left|y_{s 1}^{R}(\varrho, t)\right|>1.3,
\end{array},\right. \\
d_{13}^{R}\left(y_{s 1}^{R}(\varrho, t)\right)=\left\{\begin{array}{ll}
0.34, & \left|y_{s 1}^{R}(\varrho, t)\right| \leqslant 1.3, \\
0.13, & \left|y_{s 1}^{R}(\varrho, t)\right|>1.3,
\end{array},\right.
\end{gathered}
$$




$$
\begin{aligned}
& d_{31}^{R}\left(y_{s 3}^{R}(\varrho, t)\right)=\left\{\begin{array}{ll}
-0.18, & \left|y_{s 3}^{R}(\varrho, t)\right| \leqslant 1.3, \\
-0.26, & \left|y_{s 3}^{R}(\varrho, t)\right|>1.3,
\end{array} \quad d_{32}^{R}\left(y_{s 3}^{R}(\varrho, t)\right)= \begin{cases}0.23, & \left|y_{s 3}^{R}(\varrho, t)\right| \leqslant 1.3, \\
0.13, & \left|y_{s 3}^{R}(\varrho, t)\right|>1.3,\end{cases} \right. \\
& d_{33}^{R}\left(y_{s 3}^{R}(\varrho, t)\right)=\left\{\begin{array}{ll}
-0.15, & \left|y_{s 3}^{R}(\varrho, t)\right| \leqslant 1.3, \\
-0.24, & \left|y_{s 3}^{R}(\varrho, t)\right|>1.3,
\end{array} \quad d_{11}^{I}\left(y_{s 1}^{I}(\varrho, t)\right)= \begin{cases}0.15, & \left|y_{s 1}^{I}(\varrho, t)\right| \leqslant 1.3, \\
-0.25, & \left|y_{s 1}^{I}(\varrho, t)\right|>1.3,\end{cases} \right. \\
& d_{12}^{I}\left(y_{s 1}^{I}(\varrho, t)\right)=\left\{\begin{array}{ll}
0.38, \quad\left|y_{s 1}^{I}(\varrho, t)\right| \leqslant 1.3, \\
-0.15, \quad\left|y_{s 1}^{I}(\varrho, t)\right|>1.3,
\end{array} \quad d_{13}^{I}\left(y_{s 1}^{I}(\varrho, t)\right)= \begin{cases}0.41, & \left|y_{s 1}^{I}(\varrho, t)\right| \leqslant 1.3, \\
0.23, & \left|y_{s 1}^{I}(\varrho, t)\right|>1.3,\end{cases} \right. \\
& d_{21}^{I}\left(y_{s 2}^{I}(\varrho, t)\right)=\left\{\begin{array}{ll}
0.35, & \left|y_{s 2}^{I}(\varrho, t)\right| \leqslant 1.3, \\
0.25, & \left|y_{s 2}^{I}(\varrho, t)\right|>1.3,
\end{array} \quad d_{22}^{I}\left(y_{s 2}^{I}(\varrho, t)\right)=\left\{\begin{array}{l}
-0.35, \quad\left|y_{s 2}^{I}(\varrho, t)\right| \leqslant 1.3, \\
0.16, \quad\left|y_{s 2}^{I}(\varrho, t)\right|>1.3,
\end{array}\right.\right. \\
& d_{23}^{I}\left(y_{s 2}^{I}(\varrho, t)\right)=\left\{\begin{array}{ll}
-0.22, & \left|y_{s 2}^{I}(\varrho, t)\right| \leqslant 1.3, \\
-0.11, & \left|y_{s 2}^{I}(\varrho, t)\right|>1.3,
\end{array} \quad d_{31}^{I}\left(y_{s 3}^{I}(\varrho, t)\right)=\left\{\begin{array}{l}
0.27, \quad\left|y_{s 3}^{I}(\varrho, t)\right| \leqslant 1.3, \\
-0.16, \quad\left|y_{s 3}^{I}(\varrho, t)\right|>1.3,
\end{array}\right.\right. \\
& d_{32}^{I}\left(y_{s 3}^{I}(\varrho, t)\right)=\left\{\begin{array}{ll}
0.26, & \left|y_{s 3}^{I}(\varrho, t)\right| \leqslant 1.3, \\
0.12, & \left|y_{s 3}^{I}(\varrho, t)\right|>1.3,
\end{array} \quad d_{33}^{I}\left(y_{s 3}^{I}(\varrho, t)\right)= \begin{cases}0.36, & \left|y_{s 3}^{I}(\varrho, t)\right| \leqslant 1.3, \\
0.25, & \left|y_{s 3}^{I}(\varrho, t)\right|>1.3,\end{cases} \right. \\
& \hat{H}=\left(\begin{array}{cccccc}
-0.6 & 0.3 & 0 & 0.1 & 0.1 & 0.1 \\
0.3 & -0.9 & 0.3 & 0 & 0.1 & 0.2 \\
0 & 0.3 & -1.1 & 0.1 & 0.5 & 0.2 \\
0.1 & 0 & 0.1 & -0.5 & 0.2 & 0.1 \\
0.1 & 0.1 & 0.5 & 0.2 & -1.0 & 0.1 \\
0.1 & 0.2 & 0.2 & 0.1 & 0.1 & -0.7
\end{array}\right) .
\end{aligned}
$$

Apparently, $f_{i}^{R}(\cdot), f_{i}^{I}(\cdot), g_{i}^{R}(\cdot)$ and $g_{i}^{I}(\cdot)(i=1,2,3)$ satisfy Assumption 1 with $F_{i}^{R}=F_{i}^{I}=G_{s}^{R}=G_{s}^{I}=0.25$ and $l_{s}^{R}=l_{s}^{I}=\eta_{s}^{R}=\eta_{s}^{I}=0.25$. The input $x_{s 1}(\varrho, t)=0.3 s \sqrt{t} \cos (\pi \varrho)+i 0.2 s \sqrt{t} \cos (\pi \varrho), x_{s 2}(\varrho, t)=0.4 s \sqrt{t} \cos (\pi \varrho)+$ $i 0.7 s \sqrt{t} \cos (\pi \varrho), x_{s 3}(\varrho, t)=0.5 s \sqrt{t} \cos (\pi \varrho)+i 0.2 s \sqrt{t} \cos (\pi \varrho)$. The parameters in the controller (7) are chosen as follows: $\Upsilon^{R}=\operatorname{diag}(0.8,0.7,0.6), \Upsilon^{I}=\operatorname{diag}(0.6,1.2,0.8)$. Take $\hat{K}_{1}$ and $\hat{K}_{2}$ as follows:

$$
\hat{K}_{1}=\left(\begin{array}{ccc}
0.1 & 0.3 & 0 \\
0.2 & -0.6 & 0.1 \\
0.4 & 0 & -0.2
\end{array}\right), \hat{K}_{2}=\left(\begin{array}{ccc}
-0.2 & 0.1 & 0.3 \\
0 & -0.4 & 0.1 \\
0.5 & 0.3 & -0.6
\end{array}\right) .
$$

By exploiting the MATLAB, we can easily obtain $\rho=4.9684$ which satisfies the condition (44). And

$$
\begin{aligned}
& \lambda\left(2 I_{N} \otimes A+\hat{h} \hat{H} \otimes\left(\hat{M}+\hat{M}^{T}\right)\right)=\{0.8125,0.9246,0.9479,0.9957,1.0426,1.0726,1.0787,1.1417,1.1617,1.2000, \\
& 1.2163,1.2164,1.2514,1.2638,1.2950,1.3151,1.4000,1.4000\},
\end{aligned}
$$

which satisfies the condition (43). According to Theorem 4.1, the system (51) is passive under controller (7). Figure 3 respectively shows the evolutions of error, output and input of six nodes when the system (51) is passive. Similarly, through a simple operation based on the above parameters by using MATLAB, we have

$$
\begin{aligned}
& \lambda\left(\Psi_{1}^{R}\right)=\{-2.5350,-2.4603,-2.4500,-2.4300,-2.3988,-2.3514,-2.3329,-2.3029,-2.2767,-2.2560,-2.1849, \\
& -2.0728,-2.0456,-1.8970,-1.8619,-1.8073,-1.7243,-1.5935\}, \\
& \lambda\left(\Psi_{1}^{I}\right)=\{-2.9603,-2.8329,-2.8029,-2.7560,-2.7350,-2.6849,-2.6500,-2.6300,-2.5988,-2.5728,-2.5514, \\
& -2.4767,-1.8456,-1.6970,-1.6619,-1.6073,-1.5243,-1.3935\}, \\
& \lambda\left(2 I_{N} \otimes A+\hat{h} \hat{H} \otimes\left(\hat{M}+\hat{M}^{T}\right)\right)=\{0.8125,0.9246,0.9479,0.9957,1.0426,1.0726,1.0787,1.1417,1.1617,1.2000, \\
& 1.2163,1.2164,1.2514,1.2638,1.2950,1.3151,1.4000,1.4000\},
\end{aligned}
$$

which satisfy the condition (49). On the basis of Theorem 4.2, the system (51) is synchronized. Figure 4 depicts the simulation result of synchronization. 


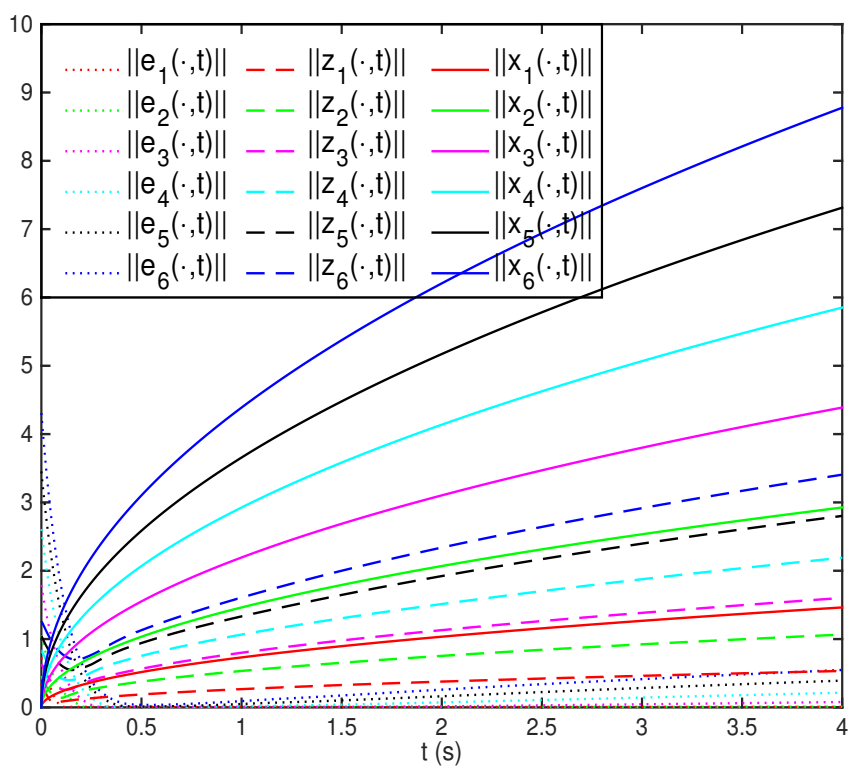

Figure 3: The norms of $e_{s}(\cdot, t), z_{s}(\cdot, t), x_{s}(\cdot, t), s=1,2, \cdots, 6$.

\section{Conclusion}

This paper has concerned with two types of CRDCVMNNs, one is with state coupling and the other is with spatial diffusion coupling. By exploiting some inequality techniques, Lyapunov functional approach as well as the construction of suitable controller, some novel criterion for ensuring passivity of these two networks have been derived. Similarly, we also have carried out some discussion on the synchronization of CRDCVMNNs with state coupling as well as spatial diffusion coupling respectively. Several simulation examples have been presented to confirm the correctness of our results at the end.

\section{Acknowledgement}

The authors would like to thank the Associate Editor and anonymous reviewers for their valuable comments and suggestions. They also wish to express their sincere appreciation to Prof. Jinliang Wang for the fruitful discussions and valuable suggestions which helped to improve this paper. This work was supported in part by the Natural Science Foundation of Tianjin City under Grants 18JCQNJC74300 and 19JCYBJC18700, in part by the National Natural Science Foundation of China under Grant 61773285 and in part by Beijing Natural Science Foundation under Grant 4202038. Dr Yang is supported in part by the Advanced Forming Research Centre (AFRC) and Lightweight Manufacturing Centre (LMC) under the Route to Impact Programme 2019-2020 funded by Innovate UK High Value Manufacturing Catapult [grant no.: AFRC_CATP_1469_R2I-Academy].

\section{References}

[1] H. Lü, W. L. He, Q. L. Han, C. Peng, Fixed-time pinning-controlled synchronization for coupled delayed neural networks with discontinuous activations, Neural Networks (2019), doi: 10.1016/j.neunet.2019.04.010.

[2] A. Chandrasekar, R. Rakkiyappan, J. Cao, Impulsive synchronization of Markovian jumping randomly coupled neural networks with partly unknown transition probabilities via multiple integral approach, Neural Networks 70 (2015) 27 - 38.

[3] W. Wu, T. Chen, Global synchronization criteria of linearly coupled neural network systems with time-varying coupling, IEEE Transactions on Neural Networks 19 (2008) 319 - 332.

[4] S. R. Lin, Y. L. Huang, S. Y. Ren, Analysis and pinning control for passivity of coupled different dimensional neural networks, Neurocomputing $321(2018) 187-200$. 


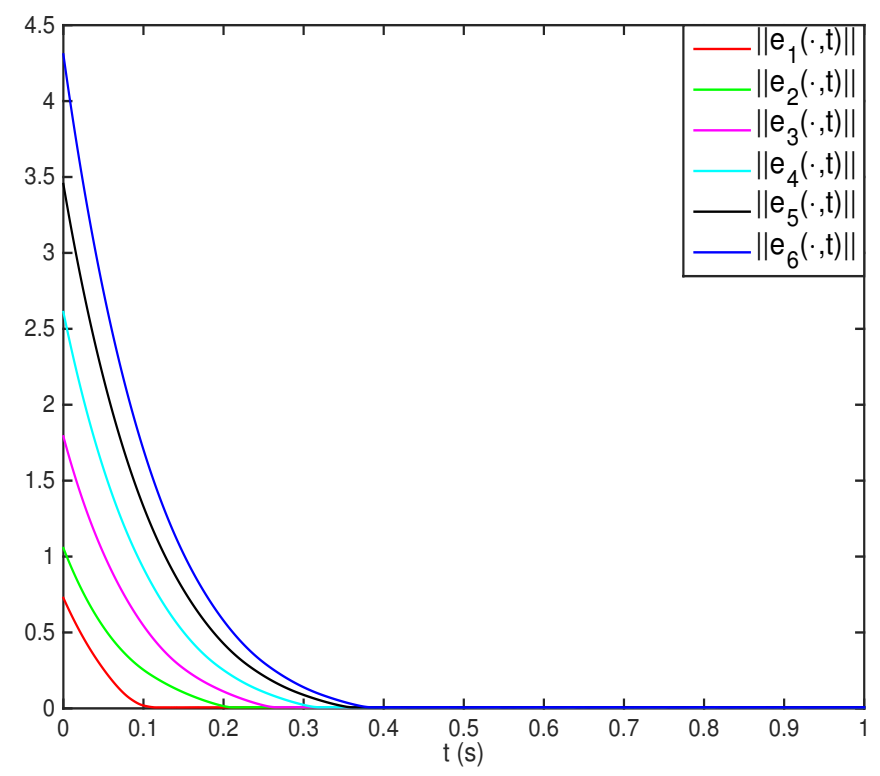

Figure 4: Time evolutions of $e_{s}(\cdot, t), s=1,2, \cdots, 6$.

[5] S. Y. Ren, J. L. Wang, J. G. Wu, Generalized passivity of coupled neural networks with directed and undirected topologies, Neurocomputing 314 (2018) $371-385$.

[6] J. Wang, L. Shen, J. W. Xia, Z. Wang, X. Y. Chen, Asynchronous dissipative filtering for nonlinear jumping systems subject to fading channels, Journal of the Franklin Institute 357 (2020) 589 - 605.

[7] J. Cheng, J.H. Park, J. Cao, W. Qi, Hidden Markov model-based nonfragile state estimation of switched neural network with probabilistic quantized outputs, IEEE Transactions on Cybernetics (2019), doi: 10.1109/TCYB.2019.2909748.

[8] J. Cheng, J.H. Park, X. Zhao, J. Cao, W. Qi, Static output feedback control of switched systems with quantization: a nonhomogeneous sojourn probability approach, International Journal of Robust and Nonlinear Control (2019), doi:10.1002/rnc.4703.

[9] J. Cheng, Y. Zhan, Nonstationary $l_{2}-l_{\infty}$ filtering for Markov switching repeated scalar nonlinear systems with randomly occurring nonlinearities, Applied Mathematics and Computation (2019), doi:10.1016/j.amc.2019.124714.

[10] X. H. Hu, J. W. Xia, Z. Wang, X. N. Song, H. Shen, Robust distributed state estimation for Markov coupled neural networks under imperfect measurements, Journal of the Franklin Institute (2020), doi: 10.1016/j.jfranklin.2020.01.021.

[11] H. Shen, S. Y. Jiao, T. W. Huang, J. D. Cao, An improved result on sampled-data synchronization of Markov jump delayed neural networks, IEEE Transactions on Systems, Man and Cybernetics: Systems (2019), doi:10.1109/TSMC.2019.2931533.

[12] H. Shen, Y. Z. Men, J. D. Cao, J. H. Park, $\mathcal{H}_{\infty}$ filtering for fuzzy jumping genetic regulatory networks with round-robin protocol: a hiddenMarkov-model-based approach. IEEE Transactions on Fuzzy Systems 28 (2020) 112 - 121.

[13] X. L. Wang, J. W. Xia, J. Wang, J Wang, Z. Wang, Passive state estimation for fuzzy jumping neural networks with fading channels based on the hidden Markov model, Physica A 535 (2019) 122437.

[14] X. H. Hu, J. W. Xia, Y. L. Wei, B. Meng, H. Shen, Passivity-based state synchronization for semi-Markov jump coupled chaotic neural networks with randomly occurring time delays, Applied Mathematics and Computation 361 (2019) 32 - 41.

[15] X. Wang, J. Xia, J. Wang, Z. Wang, J. Wang, Reachable set estimation for Markov jump LPV systems with time delays, Applied Mathematics and Computation (2020), doi:10.1016/j.amc.2020.125117.

[16] L. O. Chua, Memristor-the missing circuit element, IEEE Transactions on Circuit Theory 18 (1971) 507 - 519.

[17] H. Shen, T. Wang, J. D. Cao, G. P. Lu, Y. D. Song, T. W. Huang, Non-Fragile dissipative synchronization for markovian memristive neural networks: a gain-scheduled control scheme, IEEE Transactions on Neural Networks and Learning Systems (2018), doi: 10.1109/TNNLS.2018.2874035.

[18] L. M. Wang, Z. G. Zeng, M. F. Ge, J. H. Hu, Global stabilization analysis of inertial memristive recurrent neural networks with discrete and distributed delays, Neural Networks 105 (2018) 65 - 74.

[19] L. M. Wang, Y. Shen, G. D. Zhang, Finite-time stabilization and adaptive control of memristor-based delayed neural networks, IEEE Transactions on Neural Networks and Learning Systems 28 (2017) 2648-2659.

[20] Q. Xiao, Z. G. Zeng, Lagrange stability for T-S fuzzy memristive neural networks with time-varying delays on time scales, IEEE Transactions on Fuzzy Systems 26 (2018) 1091-1103.

[21] Y. L. Huang, S. H. Qiu, S. Y. Ren, Finite-time synchronization and passivity of coupled memristive neural networks, International Journal of Control (2019), doi: 10.1080/00207179.2019.1566640.

[22] Y. V. Pershin, M. D. Ventra, Experimental demonstration of associative memory with memristive neural networks, Neural Network 23 (2010) 
$881-886$.

[23] S. F. Yang, Z. Y. Guo, J. Wang, Robust synchronization of multiple memristive neural networks with uncertain parameters via nonlinear coupling, IEEE Transactions on Systems, Man, and Cybernetics: Systems 45 (2015) 1077 - 1086.

[24] Z. Y. Guo, S. Q. Gong, S. F. Yang, T. W. Huang, Global exponential synchronization of multiple coupled inertial memristive neural networks with time-varying delay via nonlinear coupling, Neural Networks 108 (2018) 260 - 271.

[25] Z. Y. Guo, S. F. Yang, J. Wang, Global exponential synchronization of multiple memristive neural networks with time delay via nonlinear coupling, IEEE Transactions on Neural Networks and Learning Systems 26 (2015) 1300 - 1311.

[26] R. Rakkiyappan, E. Udhaya Kumari, A. Chandrasekar, R. Krishnasamy, Synchronization and periodicity of coupled inertial memristive neural networks with supremums, Neurocomputing 214 (2016) 739 - 749.

[27] M. M. Yuan, X. Luo, W. P. Wang, L. X. Li, H. P. Peng, Pinning synchronization of coupled memristive recurrent neural networks with mixed time-varying delays and perturbations, Neural Processing Letters 49 (2019) 239 - 262.

[28] L. Shen, J. W. Xia, Y. D. Wang, X. Huang, H. Shen, HMM-based $\mathcal{H}_{\infty}$ state estimation for memristive jumping neural networks subject to fading channel, Neurocomputing (2020), doi: 10.1016/j.neucom.2020.02.016.

[29] W. Z. Chen, Y. L. Huang, S. Y. Ren, Passivity of coupled memristive delayed neural networks with fixed and adaptive coupling weights, Neurocomputing 313 (2018) 346 - 363.

[30] Y. F. Yuan, Q. K. Song, Y. R. Liu, F. E. Alsaadi, Synchronization of complex-valued neural networks with mixed two additive time-varying delays, Neurocomputing 332 (2019) 149 - 158.

[31] S. Yang, J. Yu, C. Hu, H. J. Jiang, Quasi-projective synchronization of fractional-order complex-valued recurrent neural networks, Neural Networks 104 (2018) 104 - 113.

[32] Y. Kan, J. Q. Lu, J. L. Qiu, J. G. Kurths, Exponential synchronization of time-varying delayed complex-valued neural networks under hybrid impulsive controllers, Neural Networks 114 (2019) 157 - 163.

[33] H. Zhang, X. Y. Wang, X. H. Lin, Synchronization of complex-valued neural network with sliding mode control, Journal of the Franklin Institute 353 (2016) 345 - 358

[34] H. M. Wang, S. K. Duan, T. W. Huang, L. D. Wang, C. D. Li, Exponential stability of complex-Valued memristive recurrent neural networks, IEEE Transactions on Neural Networks and Learning Systems 28 (2017) 766 - 771.

[35] D. Liu, S. Zhu, K. L. Sun, Anti-synchronization of complex-valued memristor-based delayed neural networks, Neural Networks 105 (2018) $1-13$.

[36] W. W. Zhang, H. Zhang, J. D. Cao, Fuad E. Alsaadi, D. Y. Chen, Synchronization in uncertain fractional-order memristive complex-valued neural networks with multiple time delays, Neural Networks 110 (2019) 186 - 198.

[37] R. Rakkiyappan, K. Sivaranjani, G. Velmurugan, Passivity and passification of memristor-based complex-valued recurrent neural networks with interval time-varying delays, Neurocomputing 144 (2014) 391 - 407.

[38] X. D. Li, R. Rakkiyappan, G. Velmurugan, Dissipativity analysis of memristor-based complex-valued neural networks with time-varying delays, Information Sciences 294 (2015) 645 - 665

[39] Y. L. Huang, S. X. Wang, S. Y. Ren, Pinning exponential synchronization and passivity of coupled delayed reaction-diffusion neural networks with and without parametric uncertainties, International Journal of Control (2017), doi: 10.1080/00207179.2017.1384575.

[40] Z. Qin, J. L. Wang, Q. Wang, L. J. Dai, X. Y. Guo, Passivity and synchronization of coupled reaction-diffusion neural networks with multiple coupling and uncertain inner coupling matrices, Neurocomputing 341 (2019) 26 - 40.

[41] H. A. Tang, S. K. Duan, X. F. Hu, L. D. Wang, Passivity and synchronization of coupled reaction-diffusion neural networks with multiple time-varying delays via impulsive control, Neurocomputing 318 (2018) 30 - 42.

[42] P. C. Wei, J. L. Wang, Y. L. Huang, B. B. Xu, S. Y. Ren, Impulsive control for the synchronization of coupled neural networks with reactiondiffusion terms, Neurocomputing 207 (2016) 539 - 547.

[43] B. L. Lu, H. J. Jiang, C. Hu, A. Abdurahman, Synchronization of hybrid coupled reaction-diffusion neural networks with time delays via generalized intermittent control with spacial sampled-data, Neural Networks 105 (2018) 75 - 87.

[44] J. L. Wang, H. N. Wu, L. Guo, Passivity and stability analysis of reaction-diffusion neural networks with Dirichlet boundary conditions, IEEE Transactions on neural networks, 22 (2011) 2105 - 2116.

[45] Y. L. Huang, S. Y. Ren, Passivity and passivity-based synchronization of switched coupled reaction-diffusion neural networks with state and spatial diffusion couplings, Neural Processing Letters 47 (2018) 347 - 363.

[46] K. N. Wu, B. S. Chen, Synchronization of partial differential systems via diffusion coupling, IEEE Transactions on Circuits and Systems I: Regular Papers 59 (2012) 2655 - 2668.

[47] J. L. Wang, H. N. Wu, T. W. Huang, S. Y. Ren, Pinning control strategies for synchronization of linearly coupled neural networks with reaction-diffusion terms, IEEE Transactions on Neural Networks and Learning Systems 27 (2016) 749 - 761.

[48] W. Z. Chen, Y. L. Huang, S. Y. Ren, Passivity and synchronization of coupled reaction-diffusion Cohen-Grossberg neural networks with state coupling and spatial diffusion coupling, Neurocomputing 275 (2018) 1208 - 1218.

[49] J. G. Lu, Global exponential stability and periodicity of reaction-diffusion delayed recurrent neural networks with dirichlet boundary conditions, Chaos, Solitons and Fractals 35 (2008) 116 - 125. 